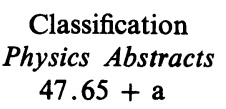

\title{
ÉTUDE THÉORIQUE ET EXPÉRIMENTALE D'UN PALIER MAGNÉTOHYDRODYNAMIQUE A CHAMP TOURNANT
}

\author{
J. BARRAL, R. BONNEFILLE et D. SCHOENHENZ \\ Laboratoire de la Chaire d'Electricité Industrielle du C.N.A.M. et \\ Laboratoire de Génie Electrique (*) des Universités de Paris-VI et Paris-XI \\ 92260 Fontenay aux Roses, France
}

(Reçu le 21 mars 1978, accepté le 20 juin 1978)

\begin{abstract}
Résumé. - On étudie le comportement d'un palier lisse à film porteur complet dont le lubrifiant, électroconducteur, est soumis à un champ magnétique tournant bipolaire. L'étude, limitée à de faibles valeurs du nombre de Reynolds modifié et des fluctuations de la vitesse, permet de mettre en évidence les caractéristiques principales du système. On montre notamment que la force portante croît avec le nombre de Hartmann. Une étude expérimentale permet de confirmer la validité de la théorie proposée, du moins tant que le régime d'écoulement est laminaire non tourbillonnaire.

Abstract. - The behaviour of a journal bearing having a complete supporting film has been studied. The electroconducting lubricant is subjected to a rotating dipolar magnetic field. The investigation, confined to low values of the modified Reynolds number and to small velocity fluctuations, allows one to point out the principal characteristics of the system. In particular, it is shown that the supporting force increases with the Hartmann number. The experiments carried out confirme the validity of the proposed theory, at least as long as the flow is purely laminar.
\end{abstract}

1. Introduction. - Les lubrifiants classiques (huiles, graisses) présentent aux hautes températures $\left(>200^{\circ} \mathrm{C}\right)$ les trois inconvénients suivants :

- Instabilité thermique.

- Tension de vapeur élevée.

- Faible conductibilité thermique.

Comme, dans les turbines et les pompes mécaniques, la température moyenne des paliers peut atteindre 200 à $300{ }^{\circ} \mathrm{C}$ (dans le domaine de l'aéronautique, on annonce des paliers devant fonctionner à plus de $500{ }^{\circ} \mathrm{C}$ ), il est nécessaire d'employer des circuits de refroidissement très importants et parfois même d'alimenter le film sous pression, afin que celle-ci soit, dans une bonne partie du film, supérieure à la pression de vapeur saturante du lubrifiant; faute de ces précautions, le lubrifiant est en moyenne partie vaporisé ou décomposé et la lubrification mauvaise. Aussi a-t-on accordé, dans les deux dernières décennies, une grande attention à la lubrification par conducteurs liquides $(\mathrm{Na}, \mathrm{K}, \mathrm{Hg}, \mathrm{Ga}, \mathrm{NaK}$, HgIn ...) en raison de leur plus grande facilité à travailler dans des conditions spécifiques difficiles.

Leur avantage essentiel est incontestablement leur haute conductivité thermique (plusieurs centaines

\footnotetext{
(*) Associé au C.N.R.S

REVUE DE PHYSIQUe APPLIQUÉE. - T. 13, № 10, OCTOBRE 1978
}

de fois celle d'une huile), ce qui assure l'absence d'échauffements locaux excessifs des pièces lubrifiées, ainsi que de grippages dus à ces échauffements; il en résulte une usure notablement moindre des surfaces portantes. Ces liquides se distinguent de plus par une tension de vapeur faible (à l'exception du mercure) et une grande stabilité thermique.

Néanmoins, un métal liquide a, en tant que lubrifiant, deux défauts :

- une viscosité cinématique faible, de l'ordre de $10^{-7}$ à $10^{-6} \mathrm{~m}^{2} / \mathrm{s}$ (par exemple, on trouve 0,5 à $0,6 \times 10^{-6} \mathrm{~m}^{2} / \mathrm{s}$ pour le sodium à $100{ }^{\circ} \mathrm{C}$ et $20 \times 10^{-6} \mathrm{~m}^{2} / \mathrm{s}$ pour une huile). Le régime de transition est donc atteint pour des vitesses modérées (quelques centaines de tr/min.) et le couple de frottement sur l'arbre devient important

- une viscosité dynamique faible $\left(10^{-3}\right.$ à $2 \times 10^{-3} \mathrm{Pl}$ ), ce qui correspond à une charge admissible faible.

L'utilisation de systèmes lubrifiés par métal liquide est donc limitée.

Pour remédier à ces inconvénients, on peut créer, dans le film, des forces volumiques, en l'occurrence, puisque le métal liquide est conducteur, des forces électromagnétiques qui aident les forces de viscosité ; on obtient ainsi des paliers lubrifiés par voie magnétohydrodynamique. 
Les premiers travaux consacrés à l'analyse de ces systèmes ont été publiés en 1962. Depuis, on compte de très nombreux articles consacrés à ce thème, la plupart présentant un caractère strictement théorique. $\mathrm{Au}$ vu de ces études, il apparaît que, par rapport à la lubrification hydrodynamique, on peut obtenir toute une série d'avantages dont, en premier lieu, une force portante supérieure. C'est ainsi que, sous l'action des forces électromagnétiques, on peut assurer la circulation du métal liquide à travers le système afin d'améliorer les conditions de refroidissement des pièces et de prévenir les échauffements locaux excessifs; la nécessité de réaliser un système spécial d'alimentation disparaît alors. De même, un fonctionnement hydrostatique est possible, sous l'action des seules forces électromagnétiques. Les insuffisances principales de la lubrification M.H.D., s'opposant à son utilisation pratique, sont liées à la structure de l'inducteur, responsable du champ magnétique; en effet, il convient de créer des champs magnétiques importants garantissant dans la veine des nombres de Hartmann élevés, avec de faibles dimensions du dispositif engendrant le champ.

Le palier M.H.D. à champ tournant, objet de l'article, est un palier lubrifié par un métal liquide soumis à un champ magnétique tournant extérieur. Le champ électromoteur, responsable des courants induits, a donc pour origine aussi bien le mouvement du liquide par rapport au champ que la variation locale de ce dernier dans le temps. On propose d'abord une formulation théorique du phénomène d'écoulement en supposant notamment négligeables l'action des forces d'inertie et les fluctuations de la vitesse dues aux forces électromagnétiques pulsantes. Après avoir étudié les variations de la résultante des forces de pression et du couple de frottement sur l'arbre pour diverses valeurs de l'induction tournante, on décrit enfin une étude expérimentale sur maquette, permettant ainsi de tester la théorie proposée.

2. Etude théorique. - 2.1 HYPOTHÈSES SIMPLIFICATRICES. - On considère, tout au long de cette étude, un palier cylindrique (Fig. 1) dont l'arbre est animé d'une vitesse angulaire constante $\Omega$ et dont la veine liquide, de géométrie définie par $a$ (épaisseur moyenne, palier centré), $r$ (rayon de l'arbre ; $a / r \ll 1$ ), $l$ (largeur de la veine; $a / l \ll 1)$ et $e$ (excentricité), est soumise, de la part du milieu extérieur, à un champ magnétique radial, bipolaire, à topographie sinusoïdale et tournant à la vitesse angulaire $\Omega$ de l'arbre. On suppose de plus que :

- le coussinet et l'arbre sont indéformables $(a, r, l$ sont constants);

- le palier est aligné ;

- les parois de la veine sont lisses et non électroconductrices ;

- le lubrifiant est un métal liquide, qui sera considéré comme un fluide bon conducteur de la chaleur, newtonien, incompressible et mouillant. De

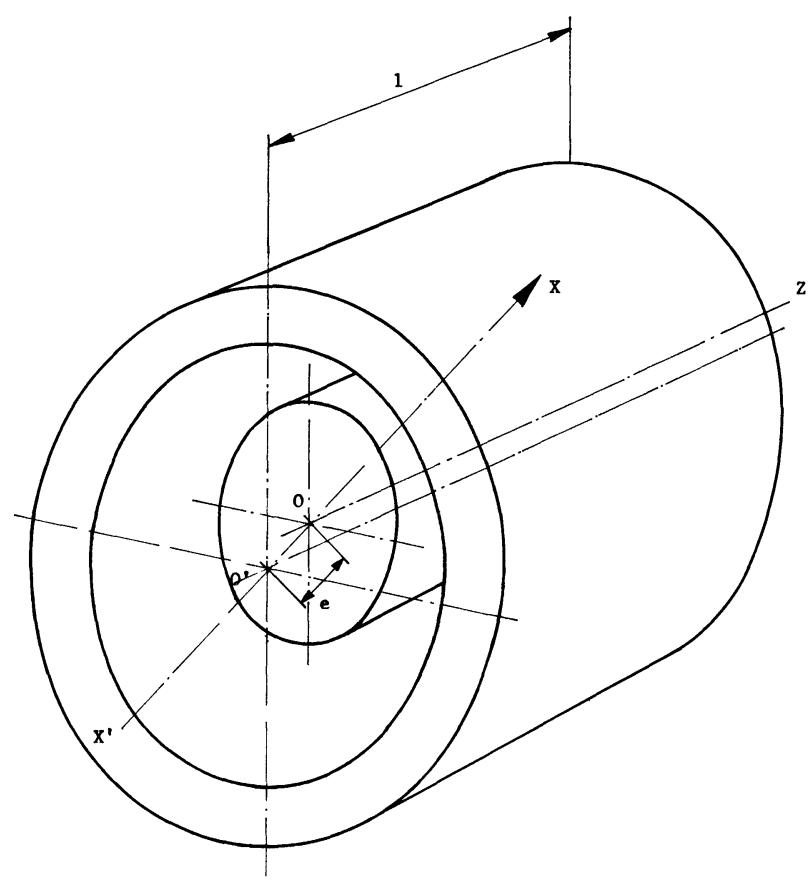

Fig. 1. - Schéma général du palier.

[Schematic representation of the bearing.]

nombreuses mesures physiques du profil de la vitesse, au voisinage de la paroi (jusqu'à $5 \times 10^{-5} \mathrm{~m}$ ) ont permis d'établir la confirmation indirecte de cette dernière hypothèse. Celle-ci est admise actuellement, même pour le mercure, d'après $C$. Fediaevski, I. Voitkounski, Y. Faddeev [17]. On désignera respectivement par $\rho$ et $\eta$ la masse volumique et la viscosité dynamique du lubrifiant, supposé non polarisable, électroconducteur et où l'effet Hall est négligeable. Sa perméabilité magnétique $\mu$ et sa permittivité diélectrique $\varepsilon$ sont donc celles du vide $\left(\mu=\mu_{0}\right.$, $\left.\varepsilon=\varepsilon_{0}\right)$ et sa conductivité électrique $\sigma$ est un scalaire ; $\rho, \eta$ et $\sigma$ sont supposés constants ;

- il n'y a pas de fuite latérale du lubrifiant (présence de joints d'étanchéité), et on néglige les phénomènes de cavitation;

- le champ magnétique extérieur, créé par un inducteur, est indépendant de la coordonnée caractérisant la largeur du palier (les effets d'extrémités sont négligés) et le nombre de Reynolds magnétique $R_{\mathrm{m}}=\mu_{0} \sigma a r \Omega$ est de valeur très faible devant l'unité : en tous points de la veine, l'induction magnétique se confond avec celle, $\vec{B}^{\text {ex }}$, due à l'inducteur seul ;

- le fonctionnement du palier est supposé stable : l'axe $Z^{\prime} Z$ de l'arbre conserve une position fixe dans le temps par rapport au coussinet. L'étude sera limitée aux faibles valeurs du nombre de Reynolds modifié $R_{\mathrm{e}}^{*}$ de l'écoulement $\left(R_{\mathrm{e}}^{*}=\frac{\rho}{\eta} \Omega a^{2}\right)-$ ce qui, dans le cas de la lubrification hydrodynamique $\left(\vec{B}^{\text {ex }}=\overrightarrow{0}\right)$ correspond à un régime d'écoulement laminaire non tourbillonnaire [18] — ainsi qu'aux faibles fluctuations de vitesse dues aux forces électromagnétiques. 
2.2 FORMULATION DU PROBLÈME. - On raisonne dans un référentiel galiléen lié au coussinet.

Le champ de vitesse $\mathbf{v}$, le champ électrique $\mathbf{E}$, la densité de courant $\mathbf{j}$ et la pression motrice $\hat{p}$, définie par

$$
\operatorname{grad} \hat{p}=\operatorname{grad} p+\rho \mathbf{g},
$$

où $p$ est la pression et $\mathbf{g}$ le champ de gravitation, vérifient, dans le cadre des approximations issues des hypothèses précédemment énoncées, les équations :

$$
\left.\begin{array}{c}
\operatorname{div} \mathbf{v}=0 \\
\rho\left(\frac{\partial \mathbf{v}}{\partial t}+\mathbf{v} \operatorname{grad} \mathbf{v}\right)=-\operatorname{grad} \hat{p}+\eta \Delta \mathbf{v}+\mathbf{j} \cdot \vec{B}^{\text {ex }} \\
\mathbf{j}=\sigma\left(\mathbf{E}+\mathbf{v} \times \vec{B}^{\text {ex }}\right) \\
\operatorname{div} \mathbf{j}=0 \\
\overleftrightarrow{\operatorname{rot} \mathbf{E}}=-\frac{\partial \vec{B}^{\text {ex }}}{\partial t}
\end{array}\right\}
$$

Les coordonnées les mieux adaptées sont les coordonnées cylindriques : un point $\mathbf{M}$ de la veine liquide est repéré par le triplet $(r+y, \theta, z)$ ou, puisque $r$ est constant, par $(y, \theta, z)$.

Les surfaces $(\theta=\mathrm{Cte})$ et $(z=\mathrm{Cte})$ sont des plans respectivement contenant et perpendiculaires à l'axe $Z^{\prime} Z$ de l'arbre. On choisit pour plan $(\theta=0)$ et $(z=0)$ les plans $(\theta=$ Cte $)$ coupant la veine en son épaisseur minimale, et $(z=\mathrm{Cte})$ coupant le palier en son milieu; la surface $(y=0)$ est la surface de l'arbre.

On note respectivement (Fig. 2) $X^{\prime} X$ l'intersection du plan $(\theta=0)$ avec le plan $(z=0)$ et $Y^{\prime} Y$ un axe tel que le trièdre $\left(X^{\prime} X, Y^{\prime} Y, Z^{\prime} Z\right)$ soit orthonormé direct.

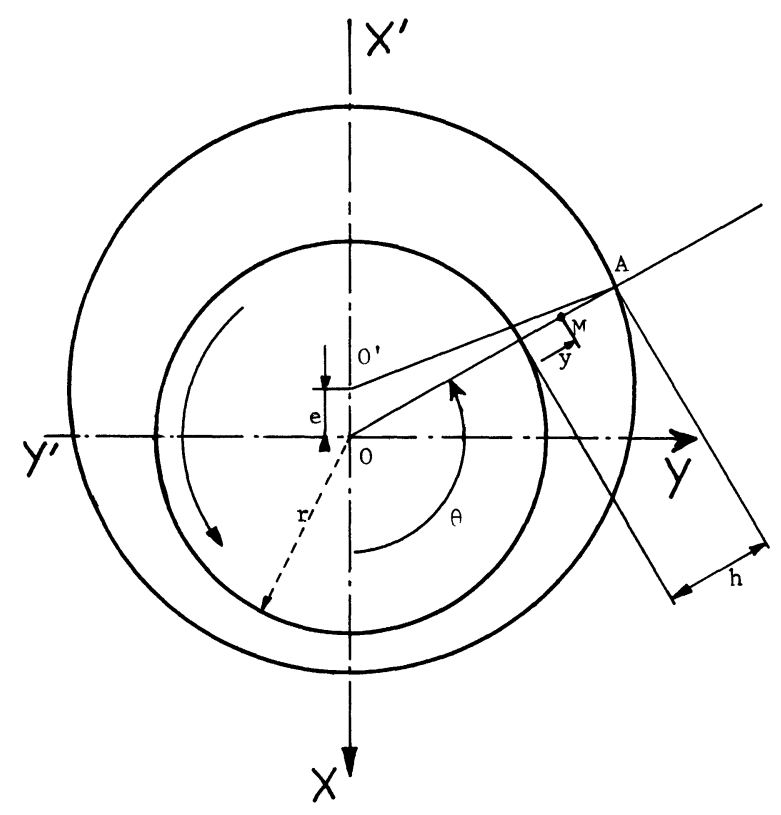

Fig. 2. - Vue en coupe du palier. [Cross-section of the bearing.]

Compte tenu de la faible valeur du rapport $\frac{a}{r}$, on peut admettre que l'induction $\vec{B}^{\text {ex }}$ ne varie pas, dans la veine, suivant $y$ et poser

$$
B_{y}^{\mathrm{ex}}=B_{0} \sqrt{2} \cos (\Omega t-\theta) ; \quad B_{\theta}^{\mathrm{ex}}=B_{z}^{\mathrm{ex}}=0
$$

où la valeur de $B_{0}$ est liée au courant électrique circulant dans l'inducteur.

Enfin, les effets d'extrémités, tant hydrauliques qu'électriques, étant négligés, on peut écrire qu'en régime établi

$$
\hat{p}_{, z}=\hat{p}_{, z}(t), \quad \mathbf{v}_{, z}=\mathbf{j}_{, z}=\mathbf{E}_{, z}=\mathbf{0} .
$$

2.2.1 Equations de l'écoulement. - Les composantes de la force électromagnétique $\mathbf{j} \times \overleftrightarrow{B^{\prime}}$ s'exerçant sur chaque particule fluide sont

$$
\left(\mathbf{j} \times \vec{B}^{\mathrm{ex}}\right)_{y}=0 ; \quad\left(\mathbf{j} \times \vec{B}^{\mathrm{ex}}\right)_{\theta}=j_{z} B_{y}^{\mathrm{ex}} ; \quad\left(\mathbf{j} \times \vec{B}^{\mathrm{ex}}\right)_{z}=-j_{\theta} . B_{y}^{\mathrm{ex}}
$$

soit, en tenant compte des expressions de $j_{\theta}$ et $j_{z}$ données par la loi d'Ohm (3)

$$
\left(\mathbf{j} \times \vec{B}^{\text {ex }}\right)_{\theta}=-\sigma v_{\theta}\left(B_{y}^{\text {ex }}\right)^{2}+\sigma E_{z} \cdot B_{y}^{\text {ex }} ; \quad\left(\mathbf{j} \times \vec{B}^{\text {ex }}\right)_{z}=-\sigma v_{z}\left(B_{y}^{\text {ex }}\right)^{2}-\sigma E_{\theta} B_{y}^{\text {ex }} .
$$

L'équation de Navier-Stokes (2) conduit alors au système

$$
\begin{array}{r}
\rho\left[\frac{\partial v_{y}}{\partial t}+v_{y} v_{y, y}+\frac{v_{\theta}}{r} \cdot v_{y, \theta}-\frac{v_{\theta}^{2}}{r}\right]=-\hat{p}_{, y}+\eta\left[v_{y, y y}+\frac{1}{r^{2}} v_{y, \theta \theta}+\frac{1}{r} v_{y, y}-\frac{2}{r^{2}} v_{\theta, \theta}-\frac{v_{y}}{r^{2}}\right] \\
\rho\left[\frac{\partial v_{\theta}}{\partial t}+v_{y} v_{\theta, y}+\frac{v_{\theta}}{r} v_{\theta, \theta}+\frac{v_{y} v_{\theta}}{r}\right]= \\
=-\hat{p}_{, \theta}+\eta\left[v_{\theta, y y}+\frac{1}{r^{2}} v_{\theta, \theta \theta}+\frac{1}{r} v_{\theta, y}+\frac{2}{r^{2}} v_{y, \theta}-\frac{v_{\theta}}{r^{2}}\right]-\sigma v_{\theta}\left(B_{y}^{\mathbf{e x}}\right)^{2}+\sigma E_{z} B_{y}^{\mathbf{e x}}
\end{array}
$$




$$
\rho\left[\frac{\partial v_{z}}{\partial t}+v_{y} v_{z, y}+\frac{v_{\theta}}{r} v_{z, \theta}\right]=-\hat{p}_{, z}+\eta\left[v_{z, y y}+\frac{1}{r^{2}} v_{z, \theta \theta}+\frac{1}{r} v_{z, y}\right]-\sigma v_{z}\left(B_{y}^{\mathbf{e x}}\right)^{2}-\sigma E_{\theta} B_{y}^{\mathbf{e x}}
$$

Soient $V_{y}^{*}$ et $V_{\theta}^{*}$ des variations caractéristiques des composantes $v_{y}$ et $v_{\theta}$ de la vitesse suivant les directions respectives de $y$ et de $\theta$. Choisissant $a$ comme longueur caractéristique suivant l'épaisseur de la veine et définissant la variable réduite

$$
\bar{y}=\frac{y}{a}
$$

l'équation de continuité (1) s'écrit

$$
\frac{V_{y}^{*}}{a}\left[\left(1+\frac{a}{r} \bar{y}\right) \bar{v}_{y}\right]_{, \bar{y}}+\frac{V_{\theta}^{*}}{r} \cdot \bar{v}_{\theta, \theta}=0 .
$$

La composante radiale $\left(\mathbf{j} \times \overrightarrow{\boldsymbol{B}}^{\mathrm{ex}}\right)_{y}$ de la force électromagnétique étant nulle, on remarque alors que l'existence d'une composante radiale $v_{y}$ de la vitesse tout comme le fait que $\theta$ est argument de $v_{\theta}$, sont dus à la seule excentricité du palier. De l'expression précédente et de la petitesse du rapport $a / r$ on déduit

$$
V_{y}^{*}=\frac{a}{r} V_{\theta}^{*} .
$$

Aux fortes excentricités, seul cas étudié ici, on peut choisir (cf. les théories de la lubrification hydrodynamique)

et par suite

$$
V_{\theta}^{*}=r \Omega
$$

$$
V_{y}^{*}=a \Omega \text {. }
$$

Désignons alors par $\tilde{V}^{*}$ une grandeur caractéristique des fluctuations de la vitesse et par $t^{*}$ un temps caractéristique de la période de ces fluctuations. Définissons les grandeurs réduites

$$
\begin{gathered}
\bar{z}=\frac{z}{r}, \quad \bar{t}=\frac{t}{t^{*}}, \quad \bar{v}_{y}=\frac{v_{y}}{a \Omega}, \quad \bar{v}_{\theta}=\frac{v_{\theta}}{r \Omega}, \quad \bar{v}_{z}=\frac{v_{z}}{r \Omega} \\
\overline{\hat{p}}=\frac{\hat{p}}{r \Omega}\left(\frac{a}{r}\right)^{2} \quad \bar{E}_{\theta}=\frac{E_{\theta}}{r \Omega B_{0}} \quad \bar{E}_{z}=\frac{E_{z}}{r \Omega B_{0}} .
\end{gathered}
$$

Les équations (6) à (8) s'écrivent sous forme réduite

$$
\begin{aligned}
& R_{\mathrm{e}}^{*} \cdot \frac{\tilde{V}^{*}}{r \Omega} \cdot \frac{1_{1}}{\Omega t^{*}} \cdot \frac{a}{r} \frac{\partial \bar{v}_{y}}{\partial \bar{t}}+R_{\mathrm{c}}^{*}\left[-\bar{v}_{\theta}^{2}+\left(\frac{a}{r}\right)^{2}\left(\bar{v}_{y} \bar{v}_{y, \bar{y}}+\bar{v}_{\theta} \bar{v}_{y, \theta}\right)\right]= \\
& =-\overline{\hat{p}}_{, y}+\left(\frac{a}{r}\right)^{2}\left[\bar{v}_{y, \bar{y} \bar{y}}+\left(\frac{a}{r}\right)\left(\bar{v}_{y, \bar{y}}-2 \bar{v}_{\theta, \theta}\right)+\left(\frac{a}{r}\right)^{2}\left(\bar{v}_{y, \theta}-\bar{v}_{y}\right)\right] \\
& R_{\mathrm{e}}^{*} \frac{\tilde{V}_{t}^{*}}{r \Omega} \cdot \frac{1}{\Omega t^{*}} \cdot \frac{\partial \bar{v}_{\theta}}{\partial \bar{t}}+R_{\mathrm{e}}^{*}\left(\bar{v}_{y} \bar{v}_{\theta, \bar{y}}+\bar{v}_{\theta} \bar{v}_{\theta, \theta}+\frac{a}{r} \bar{v}_{y} \bar{v}_{\theta}\right)= \\
& =-\overline{\hat{p}}_{, \theta}+\bar{v}_{\theta, \bar{y} \bar{y}}+\left(\frac{a}{r}\right)\left[\bar{v}_{\theta, y}+\left(\frac{a}{r}\right)\left(\bar{v}_{\theta, \theta \theta}-\bar{v}_{\theta}\right)+2\left(\frac{a}{r}\right)^{2} \bar{v}_{y, \theta}+M_{0}^{2} \times\right. \\
& \times\left\lceil\bar{E}_{z} \sqrt{2} \cos (\Omega t-\theta)-2 v_{\theta} \cos ^{2}(\Omega t-\theta)\right] \\
& R_{\mathrm{e}}^{*} \frac{\tilde{V}^{*}}{r \Omega} \frac{1}{\Omega t^{*}} \frac{\partial \bar{v}_{z}}{\partial \bar{t}}+R_{\mathrm{e}}^{*}\left(\bar{v}_{y} \bar{v}_{z, \bar{y}}+\bar{v}_{\theta} \bar{v}_{z, \theta}\right)=-\overline{\hat{p}}_{, \bar{z}}+\bar{v}_{z, \bar{y} \bar{y}}+\left(\frac{a}{r}\right)\left[\bar{l}_{z, \bar{y}}+\left(\frac{a}{r}\right) \bar{v}_{z, \theta \theta}\right]-M_{0}^{2} \times \\
& \times\left[\bar{E}_{\theta} \sqrt{2} \cos (\Omega t-\theta)+2 \bar{c}_{z} \cos ^{2}(\Omega t-\theta)\right]
\end{aligned}
$$


soit, à $\mathrm{O}(a / r)$ près

$$
\begin{aligned}
& R_{\mathrm{e}}^{*} \frac{\tilde{V}_{\mathrm{i}}^{*}}{r \Omega} \cdot \frac{1}{\Omega t^{*}} \cdot \frac{a}{r} \cdot \frac{\partial \bar{t}_{y}}{\partial \bar{t}}-R_{\mathrm{e}}^{*} \bar{v}_{\theta}^{2}=-\overline{\hat{p}}_{\bar{y}} \\
& R_{\mathrm{e}}^{*} \frac{\tilde{V}^{*}}{r \Omega} \cdot \frac{1}{\Omega t^{*}} \cdot \frac{\partial \bar{v}_{\theta}}{\partial \bar{t}}+R_{\mathrm{e}}^{*}\left(\bar{v}_{y} \bar{v}_{\theta, \bar{y}}+\bar{v}_{\theta} \bar{v}_{\theta, \theta}\right)=-\overline{\hat{p}}_{, \theta}+\bar{v}_{\theta, \bar{y} \bar{y}}+M_{0}^{2} \times \\
& \times\left[\bar{E}_{z} \sqrt{2} \cos (\Omega t-\theta)-2 \bar{v}_{\theta} \cos ^{2}(\Omega t-\theta)\right] \\
& R_{\mathrm{e}}^{*} \frac{\tilde{V}_{1}^{*}}{r \Omega} \cdot \frac{1}{\Omega t^{*}} \cdot \frac{\partial \bar{v}_{z}}{\partial \bar{t}}+R_{\mathrm{e}}^{*}\left(\bar{v}_{y} \bar{v}_{z, \bar{y}}+\bar{v}_{\theta} \bar{v}_{z, \theta}\right)=-\overline{\hat{p}}_{, \bar{z}}+\bar{v}_{z, \bar{y} \bar{y}}-M_{0}^{2} \times \\
& \times\left[\bar{E}_{\theta} \sqrt{2} \cos (\Omega t-\theta)+2 \bar{v}_{z} \cos ^{2}(\Omega t-\theta)\right]
\end{aligned}
$$

où

$$
R_{\mathrm{e}}^{*}=\frac{\rho}{\eta} \Omega a^{2} \quad \text { et } \quad M_{0}=\sqrt{\frac{\sigma}{\eta}} \cdot B_{0}^{\prime} \cdot a
$$

sont respectivement le nombre de Reynolds modifié et le nombre de Hartmann de l'écoulement. On peutévaluer $t^{*}$ à $1 / \Omega$ au voisinage du coussinet ; par contre $t^{*} \rightarrow+\infty$ près de la paroi de l'arbre où le liquide tourne au synchronisme avec le champ. En tout état de cause, $1 / \Omega t^{*}$ est, au plus de l'ordre de l'unité. Comme par hypothèse

$$
\frac{\tilde{V}^{*}}{r \Omega} \ll 1 \quad \text { et } \quad R_{\mathrm{e}}^{*} \ll 1
$$

les équations permettant la détermination du champ de vitesse et de pression prennent les formes simplifiées

$$
\begin{aligned}
& \bar{v}_{\theta, \bar{y} \bar{y}}-2 M_{0}^{2} \cos ^{2}(\Omega t-\theta) \bar{v}_{\theta}=\overline{\hat{p}}_{, \theta}-M_{0}^{2} \sqrt{2} \bar{E}_{z} \cos (\Omega t-\theta) \\
& \bar{v}_{z, \bar{y} \bar{y}}-2 M_{0}^{2} \cos ^{2}(\Omega t-\theta) \bar{v}_{z}=\overline{\hat{p}}_{, \bar{z}}+M_{0}^{2} \sqrt{2} \bar{E}_{\theta} \cos (\Omega t-\theta)
\end{aligned}
$$

où $\overline{\hat{p}}$ ne dépend que des variables $\theta, \bar{z}$ et $\bar{t}$. L'écoulement est donc caractérisé par le seul nombre de Hartmann.

De l'équation de Maxwell-Faraday (5), on déduit les équations aux dérivées partielles

$$
\left\{\begin{array}{c}
\frac{1}{r+y} E_{z, \theta}=-\frac{\partial B_{y}^{\mathrm{ex}}}{\partial t} \\
-E_{z, y}=0
\end{array}\right.
$$

puisque $\mathbf{E}$ ne dépend pas de $z$; par suite, à $\mathrm{O}(a / r)$ près

où

$$
E_{z}(\theta, t)=-r \int_{0}^{\theta} \frac{\partial B_{y}^{\text {ex }}}{\partial t} \mathrm{~d} \theta+E_{z}(0, t)
$$

$$
E_{z}(\theta, t)=+r \Omega B_{y}^{\mathrm{ex}}+E_{z}(0, t)-\left.r \Omega B_{y}^{\mathrm{ex}}\right|_{\theta=0}
$$

puisque

$$
\frac{\partial B_{y}^{\mathrm{ex}}}{\partial t}=-\Omega B_{y, \theta}^{\mathrm{ex}}
$$

Le courant $i_{z}$ traversant une section droite (section perpendiculaire à $Z^{\prime} Z$ ) de la veine a pour expression

$$
i_{z}=\int_{0}^{2 \pi} \int_{0}^{h} j_{z} \mathrm{~d} y r \mathrm{~d} \theta
$$

Par hypothèse, $i_{z}$ ne dépend pas de $z$ (et le choix de cette section droite est arbitraire), et sa valeur est nulle. Par suite et tenant compte de la loi d'Ohm

$$
\frac{1}{\sigma} i_{z}=\int_{0}^{2 \pi} \int_{0}^{h} E_{z} \mathrm{~d} y r \mathrm{~d} \theta-\int_{0}^{2 \pi} B_{y}^{\mathrm{ex}} \int_{0}^{h} v_{\theta} \mathrm{d} y r \mathrm{~d} \theta=0 .
$$


Le palier étant sans fuite, le débit-volume $q_{\mathrm{v}}$

$$
q_{\mathrm{v}}=\int_{-l / 2}^{+l / 2} \int_{0}^{h} v_{\theta} \mathrm{d} y \mathrm{~d} z=l \int_{0}^{h} v_{\theta} \mathrm{d} y
$$

ne dépend que du temps et la deuxième intégrale de l'expression (12) est nulle. On a donc

$$
\int_{0}^{2 \pi} \int_{0}^{h} E_{z} \mathrm{~d} y r \mathrm{~d} \theta=0
$$

soit, compte tenu de (11)

$$
\left[E_{z}(0,0, t)-\left.r \Omega B_{y}^{\mathrm{ex}}\right|_{\theta=0}\right] 2 \pi a r+r^{2} \Omega \int_{0}^{2 \pi} h B_{y}^{\mathrm{ex}} \mathrm{d} \theta=0
$$

et, par suite

$$
\begin{aligned}
E_{z}(0,0, t)-\left.r \Omega B_{y}^{\mathrm{ex}}\right|_{\theta=0} & =\frac{B_{0} \sqrt{2} r \Omega \varepsilon}{2 \pi} \int_{0}^{2 \pi} \cos \theta \cos (\Omega t-\theta) \mathrm{d} \theta \\
& =\frac{B_{0} r \Omega \varepsilon}{\sqrt{2}} \cos \Omega t
\end{aligned}
$$

On obtient finalement

$$
E_{z}(\theta, t)=r \Omega B_{y}^{\mathrm{ex}}+\frac{B_{0} r \Omega \varepsilon}{\sqrt{2}} \cos \Omega t
$$

ou, sous forme réduite

$$
\bar{E}_{z}(\theta, \bar{t})=\sqrt{2} \cos (\Omega t-\theta)+\frac{\varepsilon}{\sqrt{2}} \cos \Omega t
$$

L'équation (9) devient alors

$$
\bar{v}_{\theta, \bar{y} \bar{y}}-2 M_{0}^{2} \cos ^{2}(\Omega t-\theta) \bar{v}_{\theta}=\overline{\hat{p}}_{, \theta}-2 M_{0}^{2} \cos ^{2}(\Omega t-\theta)-M_{0}^{2} \varepsilon \cos \Omega t \cos (\Omega t-\theta)
$$

2.2.2 Conditions aux limites. - Le coussinet et le tourillon étant indéformables et de surface à génératrices parallèles, la géométrie de la veine est parfaitement déterminée par la seule donnée de l'épaisseur $h(\theta)$.

Pour obtenir son expression, il suffit de considérer (Fig. 2) le triangle $\mathrm{OO}^{\prime} \mathrm{A}$ pour lequel une relation classique donne

$$
(r+a)^{2}=e^{2}+(r+h)^{2}+2 e(r+h) \cos \theta
$$

soit à $\mathrm{O}\left(\frac{a}{r}\right)^{2}$ près

$$
h=a(1-\varepsilon \cos \theta)
$$

en définissant l'excentricité relative $\varepsilon=\frac{e}{a}$.

En posant

$$
\bar{h}=\frac{h}{a}=1-\varepsilon \cos \theta
$$

les conditions d'adhérence s'écrivent alors :

$$
\forall t \forall \theta\left\{\begin{array}{lll}
\bar{y}=0 & \bar{v}_{\theta}=1 & \bar{v}_{y}=\bar{v}_{z}=0 \\
\bar{y}=\bar{h} & \bar{v}_{\theta}=0 & \bar{v}_{y} \approx \bar{v}_{z}=0
\end{array}\right.
$$

2.2.3 Grandeurs globales. - Les grandeurs globales, caractéristiques d'un palier, sont, pour l'essentiel, sa force portante $\mathbf{F}$, égale et opposée à la charge exercée par l'extérieur sur l'arbre, et le couple de frottement $C$ exercé par le lubrifiant sur l'arbre. Les composantes de $\mathbf{F}$ suivant les trois axes $X^{\prime} X$, $Y Y^{\prime}$ et $Z Z^{\prime}$ ont pour expressions

$$
\left\{\begin{array}{l}
F_{X}=-\int_{-l / 2}^{l / 2} \int_{0}^{2 \pi}\left[\hat{p}-\frac{\left(B_{y}^{\text {ex }}\right)^{2}}{2 \mu_{0}}\right] r \mathrm{~d} \theta \cos \theta \mathrm{d} z \\
F_{Y}=-\int_{-l / 2}^{l / 2} \int_{0}^{2 \pi}\left[\hat{p}-\frac{\left(B_{y}^{\text {ex }}\right)^{2}}{2 \mu_{0}}\right] r \mathrm{~d} \theta \sin \theta \mathrm{d} z \\
F_{Z}=0
\end{array}\right.
$$


soit, en tenant compte de l'expression proposée pour $B_{y}^{\text {ex }}$

$$
\left\{\begin{array}{l}
F_{X}=-\int_{-l / 2}^{l / 2} \int_{0}^{2 \pi} \hat{p} \cos \theta \cdot r \mathrm{~d} \theta \mathrm{d} z \\
F_{Y}=-\int_{-l / 2}^{l / 2} \int_{0}^{2 \pi} \hat{p} \sin \theta \cdot r \mathrm{~d} \theta \mathrm{d} z \\
F_{Z}=0 .
\end{array}\right.
$$

Après une intégration par parties et en utilisant la pression motrice réduite $\overline{\hat{p}}$, ces expressions deviennent, puisque $\overline{\hat{p}}_{, \theta}$ ne dépend pas de $\bar{z}$, sous forme réduite

$$
\begin{aligned}
& \bar{F}_{X}=\int_{0}^{2 \pi} \overline{\hat{p}}_{, \theta} \sin \theta \mathrm{d} \theta \\
& \bar{F}_{Y}=-\int_{0}^{2 \pi} \overline{\hat{p}}_{, \theta} \cos \theta \mathrm{d} \theta
\end{aligned}
$$

en posant

$$
\left(\bar{F}_{X}, \bar{F}_{Y}\right)=\frac{a^{2}}{\eta r^{3} l \Omega}\left(F_{X}, F_{Y}\right)
$$

On définit le couple de frottement par

$$
C=\int_{0}^{2 \pi}\left[\left.\eta v_{\theta, y}\right|_{y=0} r l\right] \mathrm{d} \theta
$$

soit, sous forme réduite

$$
\bar{C}=\left.\int_{0}^{2 \pi} \bar{v}_{\theta, \bar{y}}\right|_{\bar{y}=0} \mathrm{~d} \theta
$$

$$
\bar{C}=\frac{a^{2}}{\eta \Omega r^{3} l} C
$$

2.3 DÉTERmination DES GRANDEURS GLOBALES. Toutes ces grandeurs se calculent à partir du couple $\left(\bar{v}_{\theta}, \overline{\hat{p}}_{\theta}\right)$, dont la détermination exige l'intégration, compte tenu des conditions aux limites énoncées précédemment, de l'équation aux dérivées partielles (14). Cette intégration s'avère particulièrement difficile du fait que, a priori, $\bar{v}_{\theta}$ et $\overline{\hat{p}}$ dépendent non seulement de $\theta$, de $\bar{y}$ (pour $\bar{v}_{\theta}$ ) et de $\bar{z}$ (pour $\overline{\hat{p}}$ ), mais aussi du temps.

Le problème se simplifie si l'on remarque que le fonctionnement du palier est stable, pour une charge donnée, tant que $\mathbf{F}$ reste, au cours du temps, à peu près constant, autrement dit, tant que $F_{X}$ et $F_{Y}$ prennent des valeurs voisines de leurs moyennes temporelles $\left\langle F_{X}\right\rangle$ et $\left\langle F_{Y}\right\rangle$ effectuées sur une période $2 \pi / \Omega$.

Utilisant l'expression de $\overline{\hat{p}}_{, \theta}$ fournie par l'équation (14), on constate, conformément à nos hypothèses, qu'il faut pour cela que le champ des vitesses subisse de la part de la force électromagnétique pulsante, des fluctuations d'amplitude faible devant $\left\langle v_{\theta}\right\rangle$, le degré d'approximation étant le même dans l'assimilation de $\bar{F}_{X}\left(\right.$ ou $\left.\bar{F}_{Y}\right)$ à $\left\langle\bar{F}_{X}\right\rangle$ (ou $\left.\left\langle\bar{F}_{Y}\right\rangle\right)$ que dans celle de $\bar{v}_{\theta}$ à $\left\langle\bar{v}_{\theta}\right\rangle$.

En désignant par

$$
\overline{\widetilde{v}}_{\theta}=\frac{\tilde{v}_{\theta}}{\tilde{V}^{*}}
$$

la fluctuation de la vitesse $\bar{v}_{\theta}$, cette dernière s'écrit

$$
\bar{v}_{\theta}=\left\langle\bar{v}_{\theta}\right\rangle+\overline{\widetilde{v}}_{\theta}
$$

et l'équation (14) devient, après avoir pris la moyenne temporelle de chaque terme

$$
\left\langle\bar{v}_{\theta}\right\rangle_{, \bar{y} \bar{y}}-M_{0}^{2}\left\langle\bar{v}_{\theta}\right\rangle_{.}-M_{0}^{2}\left\langle\overline{\tilde{v}}_{\theta} \cos r(\Omega t-\theta)\right\rangle=\langle\overline{\hat{p}}\rangle_{, \theta}-M_{0}^{2}-M_{0}^{2} \frac{\varepsilon}{2} \cos \theta
$$

soit à $\mathrm{O}\left(\tilde{V}^{*} / r \Omega\right)$ près

$$
\left\langle\bar{v}_{\theta}\right\rangle_{, \bar{y} \bar{y}}-M_{0}^{2}\left\langle\bar{v}_{\theta}\right\rangle=\langle\overline{\hat{p}}\rangle_{. \theta}-M_{0}^{2}-M_{0}^{2} \frac{\varepsilon}{2} \cos \theta
$$

Comme $\langle\overline{\hat{p}}\rangle$ ne dépend pas de $\bar{y}$, l'intégration, par rapport à cette variable, compte tenu des conditions d'adhérence énoncées en 2.2 .2 conduit pour $\left\langle\bar{v}_{\theta}\right\rangle$ à l'expression

$$
\left\langle\bar{v}_{\theta}\right\rangle=\frac{\sinh M_{0}(\bar{h}-\bar{y})}{\sinh M_{0} \bar{h}}+\frac{1}{M_{0}^{2}}\left[\langle\overline{\hat{p}}\rangle_{. \theta}-M_{0}^{2}-M_{0}^{2} \frac{\varepsilon}{2} \cos \theta\right]\left[\frac{\sinh M_{0}(\bar{h}-\bar{y})+\sinh M_{0} \bar{y}}{\sinh M_{0} \bar{h}}-1\right] .
$$

Une deuxième intégration par rapport à $\bar{y}$ permet de calculer le débit réduit $\bar{q}_{\mathrm{v}}$

$$
\bar{q}_{\mathrm{v}}=\int_{0}^{\bar{h}}\left\langle\bar{v}_{\theta}\right\rangle \mathrm{d} \bar{y}=\frac{\cosh M_{0} \bar{h}-1}{M_{0} \sinh M_{0} \bar{h}}+\frac{1}{M_{0}^{2}}\left[\langle\overline{\hat{p}}\rangle_{, \theta}-M_{0}^{2}-M_{0}^{2} \frac{\varepsilon}{2} \cos \theta\right]\left[2 \frac{\cosh M_{0} \bar{h}-1}{M_{0} \sinh M_{0} \bar{h}}-\bar{h}\right]
$$


On en déduit

$$
\langle\overline{\hat{p}}\rangle_{, \theta}=-\bar{q}_{\mathrm{v}} F_{1}(\theta)-F_{2}(\theta)+M_{0}^{2}+M_{0}^{2} \frac{\varepsilon}{2} \cos \theta
$$

en posant

$$
F_{1}(\theta)=\frac{M_{0}^{3} \sinh M_{0} \bar{h}}{M_{0} \bar{h} \sinh M_{0} \bar{h}-2\left(\cosh M_{0} \bar{h}-1\right)}, \quad F_{2}(\theta)=\frac{M_{0}^{2}\left(1-\cosh M_{0} \bar{h}\right)}{M_{0} \bar{h} \sinh M_{0} \bar{h}-2\left(\cosh M_{0} \bar{h}-1\right)}
$$

Intégrons (19) par rapport à $\theta$ sur l'intervalle $[0,2 \pi]$; il vient

avec

$$
0=-\bar{q}_{\mathrm{v}} I_{1}-I_{2}+2 \pi M_{0}^{2}
$$

On en tire

$$
I_{1}=\int_{0}^{2 \pi} F_{1}(\theta) \mathrm{d} \theta, \quad I_{2}=\int_{0}^{2 \pi} F_{2}(\theta) \mathrm{d} \theta
$$

$$
\bar{q}_{\mathrm{v}}=-\frac{I_{2}-2 \pi M_{0}^{2}}{I_{1}}
$$

On obtient ainsi les expressions

$$
\begin{aligned}
\langle\overline{\hat{p}}(\theta, t)\rangle-\langle\overline{\hat{p}}(0, t)\rangle & =-\bar{q}_{\mathrm{v}} \int_{0}^{\theta} F_{1}(\theta) \mathrm{d} \theta-\int_{0}^{\theta} F_{2}(\theta) \mathrm{d} \theta+M_{0}^{2} \theta+M_{0}^{2} \frac{\varepsilon}{2} \sin \theta \\
\left\langle\bar{F}_{X}\right\rangle & =-\bar{q}_{\mathrm{v}} X_{1}^{\prime}-X_{2}^{\prime}=\frac{I_{2}}{I_{1}} X_{1}^{\prime}-X_{2}^{\prime}-2 \pi M_{0}^{2} \frac{X_{1}^{\prime}}{I_{1}} \\
\left\langle\bar{F}_{Y}\right\rangle & =\bar{q}_{\mathrm{v}} X_{1}+X_{2}=-\frac{I_{2}}{I_{1}} X_{1}+X_{2}+2 \pi M_{0}^{2} \frac{X_{1}}{I_{1}}-M_{0}^{2} \frac{\pi}{2} \varepsilon
\end{aligned}
$$

après avoir noté

$$
\begin{array}{ll}
X_{1}=\int_{0}^{2 \pi} F_{1}(\theta) \cos \theta \mathrm{d} \theta, & X_{2}=\int_{0}^{2 \pi} F_{2}(\theta) \cos \theta \mathrm{d} \theta \\
X_{1}^{\prime}=\int_{0}^{2 \pi} F_{1}(\theta) \sin \theta \mathrm{d} \theta, & X_{2}^{\prime}=\int_{0}^{2 \pi} F_{2}(\theta) \sin \theta \mathrm{d} \theta
\end{array}
$$

$F_{1}(\theta)$ et $F_{2}(\theta)$ sont des fonctions paires et périodiques (période $2 \pi$ ) de $\theta$. On en déduit que les intégrales $X_{1}^{\prime}$ et $X_{2}^{\prime}$, et, par suite, la composante $\bar{F}_{X}$, sont nulles; la force de pression F est donc orientée suivant $Y^{\prime} Y$ (Fig. 3).

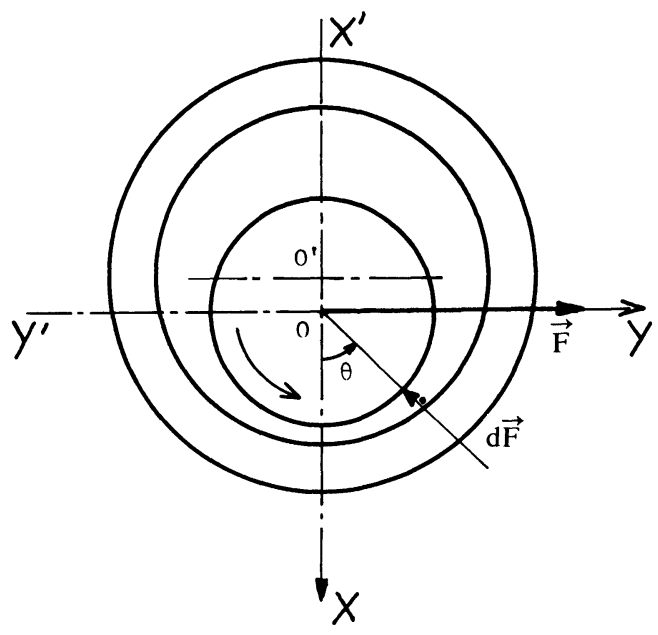

Fig. 3. - Répartition des forces de pression : dF force élémentaire; $\mathbf{F}$ force résultante.

[Distribution of the pressure forces : dF, elementary force; $\mathbf{F}$ resulting force.] 
Dérivant par rapport à $\bar{y}$ l'expression (18) de $\left\langle v_{\theta}\right\rangle$, on obtient

$$
\left.\left\langle\bar{v}_{\theta}\right\rangle_{, \bar{y}}\right|_{\bar{y}=0}=-\frac{M_{0}}{\tanh M_{0} \bar{h}}+\frac{\left(1-\cosh M_{0} \bar{h}\right)}{M_{0} \sinh M_{0} \bar{h}}\left(\langle\overline{\hat{p}}\rangle_{, \theta}-M_{0}^{2}-M_{0}^{2} \frac{\varepsilon}{2} \cos \theta\right)
$$

soit, compte tenu de la relation (18)

$$
\left.\left\langle\bar{v}_{\theta}\right\rangle_{, \bar{y}}\right|_{\bar{y}=0}=-\frac{M_{0}}{\tanh M_{0} \bar{h}}-\frac{\left(1-\cosh M_{0} \bar{h}\right)}{M_{0} \sinh M_{0} \bar{h}}\left(F_{2}(\theta)+\bar{q}_{\mathrm{v}} F_{1}(\theta)\right)
$$

Le couple réduit $\bar{C}$ devient alors, d'après (17)

où

$$
\bar{C}=-\int_{0}^{2 \pi} \frac{M_{0}}{\tanh M_{0} \bar{h}} \mathrm{~d} \theta-\int_{0}^{2 \pi} \bar{q}_{\mathrm{v}} F_{2}(\theta) \mathrm{d} \theta-\int_{0}^{2 \pi} F_{2}(\theta) \frac{1-\cosh M_{0} \bar{h}}{M_{0} \sinh M_{0} \bar{h}} \mathrm{~d} \theta
$$

$$
\bar{C}=-L_{3}-L_{2}-\bar{q}_{\mathrm{v}} I_{2}=-L_{3}-L_{2}+\frac{I_{2}^{2}}{I_{1}}-2 \pi M_{0}^{2} \frac{I_{2}}{I_{1}}
$$

en posant

$$
L_{2}=\int_{0}^{2 \pi} F_{2}(\theta) \cdot \frac{1-\cosh M_{0} \bar{h}}{M_{0} \sinh M_{0} \bar{h}} \mathrm{~d} \theta, \quad L_{3}=\int_{0}^{2 \pi} \frac{M_{0}}{\tanh M_{0} \bar{h}} \mathrm{~d} \theta
$$

Les figures $4,5 a$ et $5 b$ représentent respectivement les évolutions (d'après traitement numérique sur ordinateur) de l'accroissement

$$
\Delta\langle\overline{\hat{p}}\rangle=\langle\overline{\hat{p}}(\theta, \bar{z}, \bar{t})\rangle-\langle\overline{\hat{p}}(0, \bar{z}, \bar{t})\rangle
$$

en fonction de $\theta$, pour diverses valeurs de $M_{0}^{2}$ et pour une excentricité relative de 0,8 et des rapports

$$
\frac{\bar{F}_{Y}}{\left[\bar{F}_{Y}\right]_{M_{0}=0}} \quad \text { et } \frac{\bar{C}}{[\bar{C}]_{M_{0}=0}}
$$

pour différentes valeurs de $M_{0}^{2}$ et de $\varepsilon$.

Nous constatons que l'effet M.H.D. améliore la répartition de la pression dans la veine liquide.

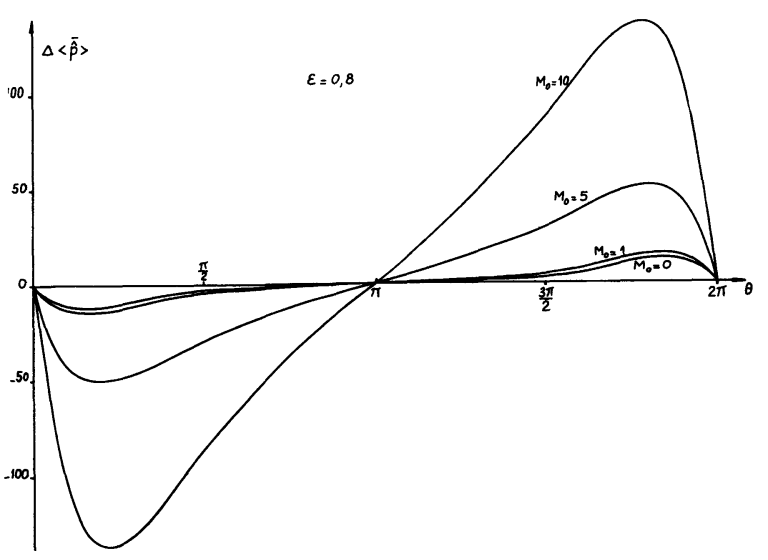

Fig. 4. - Accroissement des pressions moyennes en fonction de l'angle polaire.

[Increase of the mean pressures as a function of the polar angle.]
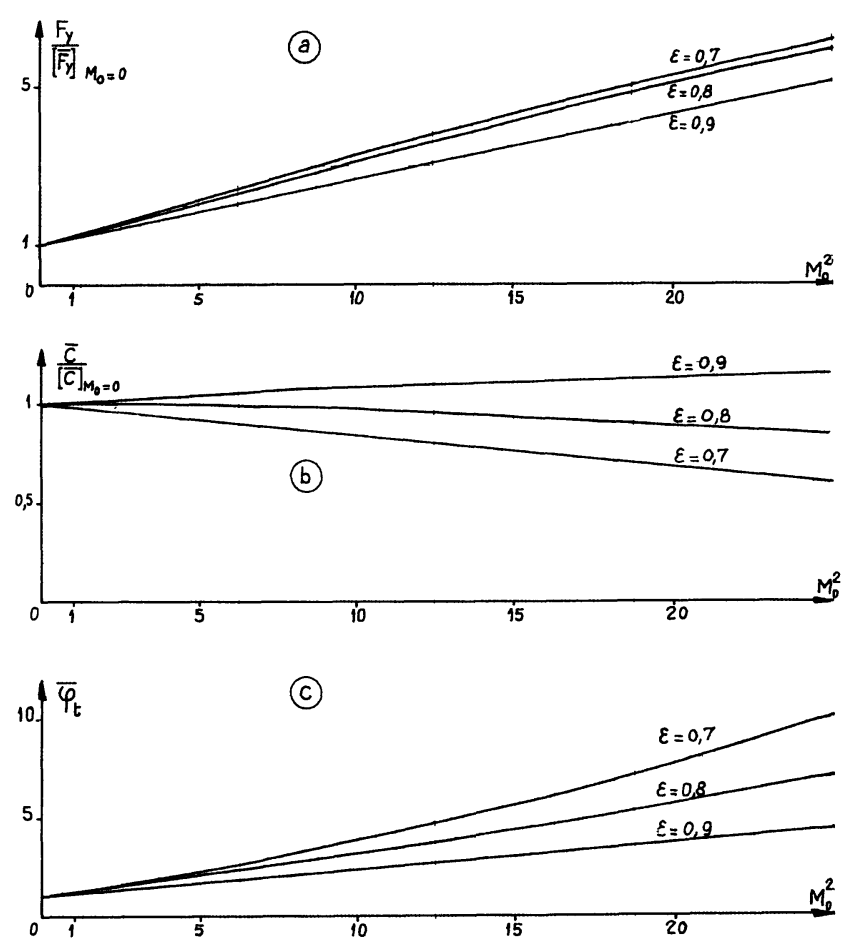

Fig. 5. - Variations du rapport $\frac{\bar{F}_{Y}}{\left[\bar{F}_{Y}\right]_{M_{0}=0}}(a)$, du rapport $\frac{\bar{C}}{[\bar{C}]_{M_{0}=0}} \quad(b)$ et du coefficient de transfert $\bar{\varphi}_{\mathrm{t}}(c)$, en fonction du carré du nombre de Hartmann et pour plusieurs valeurs de l'excentricité $\varepsilon$.

[Variations of the ratio $\frac{\bar{F}_{Y}}{\left[\bar{F}_{Y^{\prime}}\right]_{\underline{M}_{0}=0}}(a)$, of the ratio $\frac{\bar{C}}{[\bar{C}]_{M_{0}=0}}(b)$, and of the transfert coefficient $\bar{\varphi}_{\mathrm{t}}(c)$ as a function of the Hartmann number squared, for various excentricity $\varepsilon$ values.] 
Nous remarquons également la faible influence des expressions hyperboliques en $M_{0}$ sur la force portante $\mathbf{F}$ et sur le couple $C$. Il est possible d'admettre, en première approximation, que les variations de $\bar{F}_{Y}$ et $\bar{C}$ sont proportionnelles au carré du nombre de Hartmann. La figure $5 b$ montre qu'il existe une valeur frontière de l'excentricité relative $\varepsilon$, comprise entre 0,8 et 0,9 pour laquelle le couple réduit $\bar{C}$ diminue lorsque $M_{0}$ croît.

2.4 COEFFiCIENT DE TRANSFERT. - L'introduction de la notion de rendement du palier M.H.D. étudié pose un problème délicat, car la puissance électrique qui lui est fournie est, finalement, entièrement convertie en pertes. Ces dernières n'ont d'ailleurs qu'une importance relative devant la puissance absorbée par la machine qui utilise de tels paliers.

On a été ainsi amené à introduire [20] un coefficient de transfert $\varphi_{\mathrm{t}}$ homogène à l'inverse d'une vitesse, et défini par

$$
\varphi_{\mathrm{t}}=\frac{\|\mathbf{F}\|}{C \Omega}
$$

ou, compte tenu des résultats du paragraphe précédent

$$
\varphi_{\mathrm{t}}=\frac{\bar{F}_{y}}{\bar{C}} \cdot \frac{1}{a \Omega} .
$$

L'effet M.H.D. est d'autant plus efficace que $\varphi_{\mathrm{t}}$ est plus grand. Nous avons représenté, sur la figure $5 c$, les variations, en fonction de $M_{0}^{2}$ et de $\varepsilon$, du rapport.

$$
\bar{\varphi}_{\mathrm{t}}=\frac{\varphi_{\mathrm{t}}}{\left[\varphi_{\mathrm{t}}\right]_{M_{0}=0}} .
$$

On constate que $\bar{\varphi}_{\mathrm{t}}$ croît avec $M_{0}$ mais décroît, pour une valeur de $M_{0}$ donnée, lorsque $\varepsilon$ croît, c'est-à-dire quand la charge des paliers augmente.

2.5 Domaine De validité. - L'intégration de l'équation (14), non moyennée dans le temps, compte tenu des conditions d'adhérence, conduit à l'expression suivante de la vitesse

$$
\begin{aligned}
\bar{c}_{\theta}=\left[\frac{M^{\prime}\left(2 \pi M_{0}^{2}-I_{2}^{\prime}\right)}{I_{1}^{\prime} 2\left[\left(1-\cosh M^{\prime} \bar{h}\right)+M^{\prime} \bar{h} \sinh M^{\prime} \bar{h}\right]}+\frac{1}{2 \sinh M^{\prime} \bar{h}-M^{\prime} \bar{h}\left(1+\cosh M^{\prime} \bar{h}\right)}\right] \times \\
\times\left[\left(1-\cosh M^{\prime} \bar{y}\right) \sinh M^{\prime} \bar{h}-(1-\cosh M \bar{h}) \sinh M^{\prime} \bar{y}\right]+\cosh M^{\prime} \bar{y}-\frac{\sinh M^{\prime} \bar{y}}{\tanh M^{\prime} \bar{h}}
\end{aligned}
$$

avec

$$
\begin{aligned}
M^{\prime} & =M_{0} \sqrt{2} \cos (\Omega t-\theta) \\
I_{1}^{\prime} & =\int_{0}^{2 \pi} \frac{M^{\prime 3} \sinh M^{\prime} \bar{h}}{2\left(1-\cosh M^{\prime} \bar{h}\right)+M^{\prime} \bar{h} \sinh M^{\prime} \bar{h}} \mathrm{~d} \theta \\
I_{2}^{\prime} & =\int_{0}^{2 \pi} \frac{M^{\prime 2}\left(1-\cosh M^{\prime} \bar{h}\right)}{2\left(1-\cosh M^{\prime} \bar{h}\right)+M^{\prime} \bar{h} \sinh M^{\prime} \bar{h}} \mathrm{~d} \theta .
\end{aligned}
$$

Il faut remarquer que les intégrales $I_{1}^{\prime}$ et $I_{2}^{\prime}$ n'admettent pas une solution analytique; aussi avonsnous procédé à une intégration numérique sur ordinateur.

Les figures $6 a$ à $6 d$ donnent un exemple de la variation de $\underline{v}_{\theta}$ en fonction de l'angle polaire $\Omega t \operatorname{pour} \theta=0$ et $\bar{y}=\frac{\bar{h}(0)}{2}$.

On constate, d'après l'allure des courbes, que pour des valeurs du nombre de Hartmann n'excédant pas $3 \sqrt{2}$, l'amplitude de la fluctuation de la vitesse ne dépasse pas $10 \%$ de la valeur moyenne $\left\langle\bar{v}_{\theta}\right\rangle$. De plus, des calculs numériques sur ordinateur ont montré que, pour

$$
0,8 \leqslant \varepsilon<1, \quad 0 \leqslant M_{0} \leqslant 3 \sqrt{2}
$$

les fonctions

$$
\begin{aligned}
& F_{1}^{\prime}(\theta)=\frac{M^{\prime 3} \sinh M^{\prime} \bar{h}}{2\left(1-\cosh M^{\prime} \bar{h}\right)+M^{\prime} \bar{h} \sinh M^{\prime} \bar{h}} \\
& F_{2}^{\prime}(\theta)=\frac{M^{2}\left(1-\cosh M^{\prime} \bar{h}\right)}{2\left(1-\cosh M^{\prime} \bar{h}\right)+M^{\prime} \bar{h} \sinh M^{\prime} \bar{h}}
\end{aligned}
$$

sont des fonctions paires de $\theta$, périodiques (de période $2 \pi$ ) et à valeurs pratiquement indépendantes de $\Omega t$, avec une précision supérieure à $6 \%$ pour $F_{1}^{\prime}(\theta)$ et $0,04 \%$ pour $F_{2}^{\prime}(\theta)$. On en déduit alors, en reprenant un raisonnement fait auparavant, que les intégrales

$$
X_{1}^{\prime \prime}=\int_{0}^{2 \pi} F_{1}^{\prime}(\theta) \sin \theta \mathrm{d} \theta \quad X_{2}^{\prime \prime}=\int_{0}^{2 \pi} F_{2}^{\prime}(\theta) \sin \theta \mathrm{d} \theta
$$



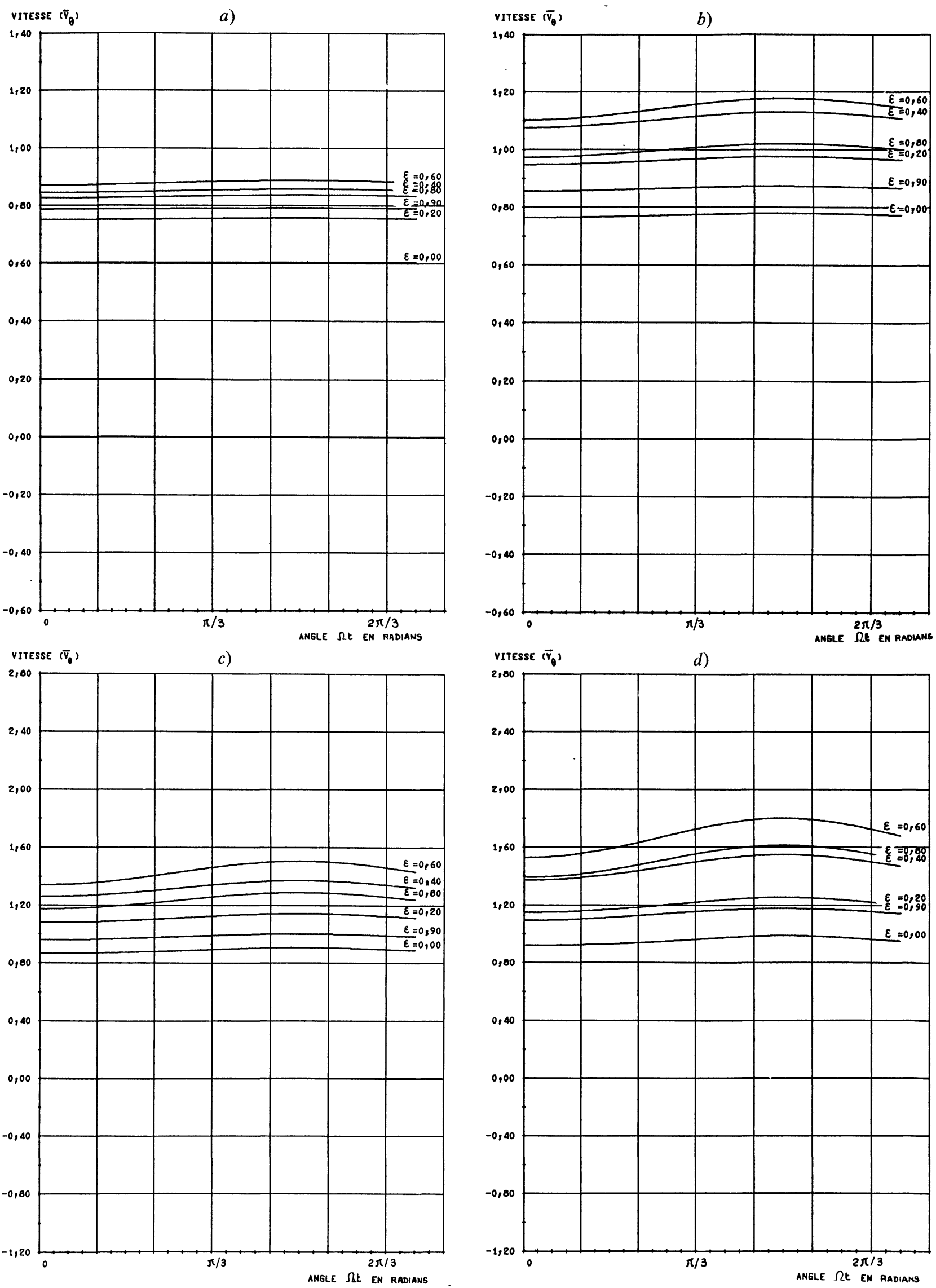

Fig. 6. - Vitesse $\bar{v}_{\theta}$, en fonction de $\Omega t$, pour différentes valeurs de l'excentricité $\left.\varepsilon: a) M_{0}=1,41, \bar{y}=0,5 \bar{h}, \theta=0 ; b\right) M_{0}=2,83$, $\left.\bar{y}=0,5 \bar{h}, \theta=0 ; c) M_{0}=4,24, \bar{y}=0,5 \bar{h}, \theta=0 ; d\right) M_{0}=5,66$, $\bar{y}=0,5 \bar{h}, \theta=0$.

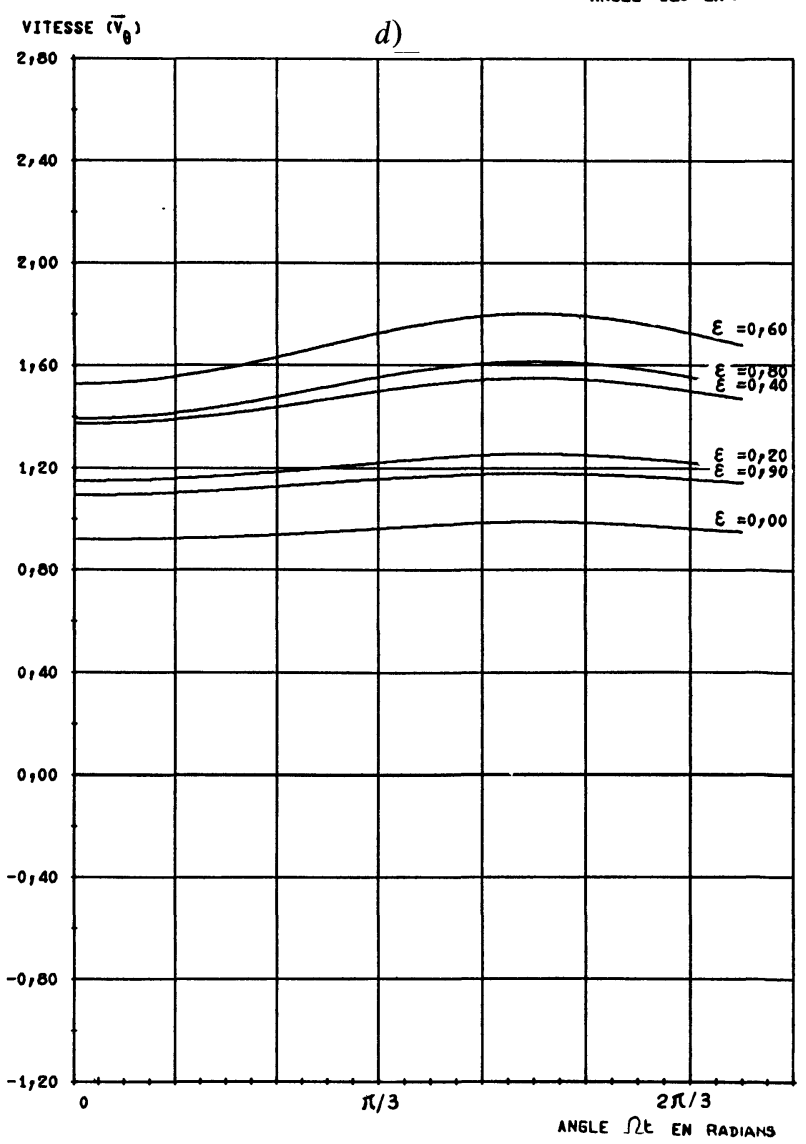

[Velocity $\bar{v}_{\theta}$, versus $\Omega t$, for different values of the excentricity $\varepsilon$ : a) $\left.\cdot M_{0}=1.41, \bar{y}=0.5 \quad \bar{h}, \theta=0 ; b\right) M_{0}=2.83, \bar{y}=0.5 \bar{h}$, $\left.\theta=0 ; c) M_{0}=4.24, \bar{y}=0.5 \bar{h}, \theta=0 ; d\right) M_{0}=5.66, \bar{y}=0.5 \bar{h}$, $\theta=0$.] 

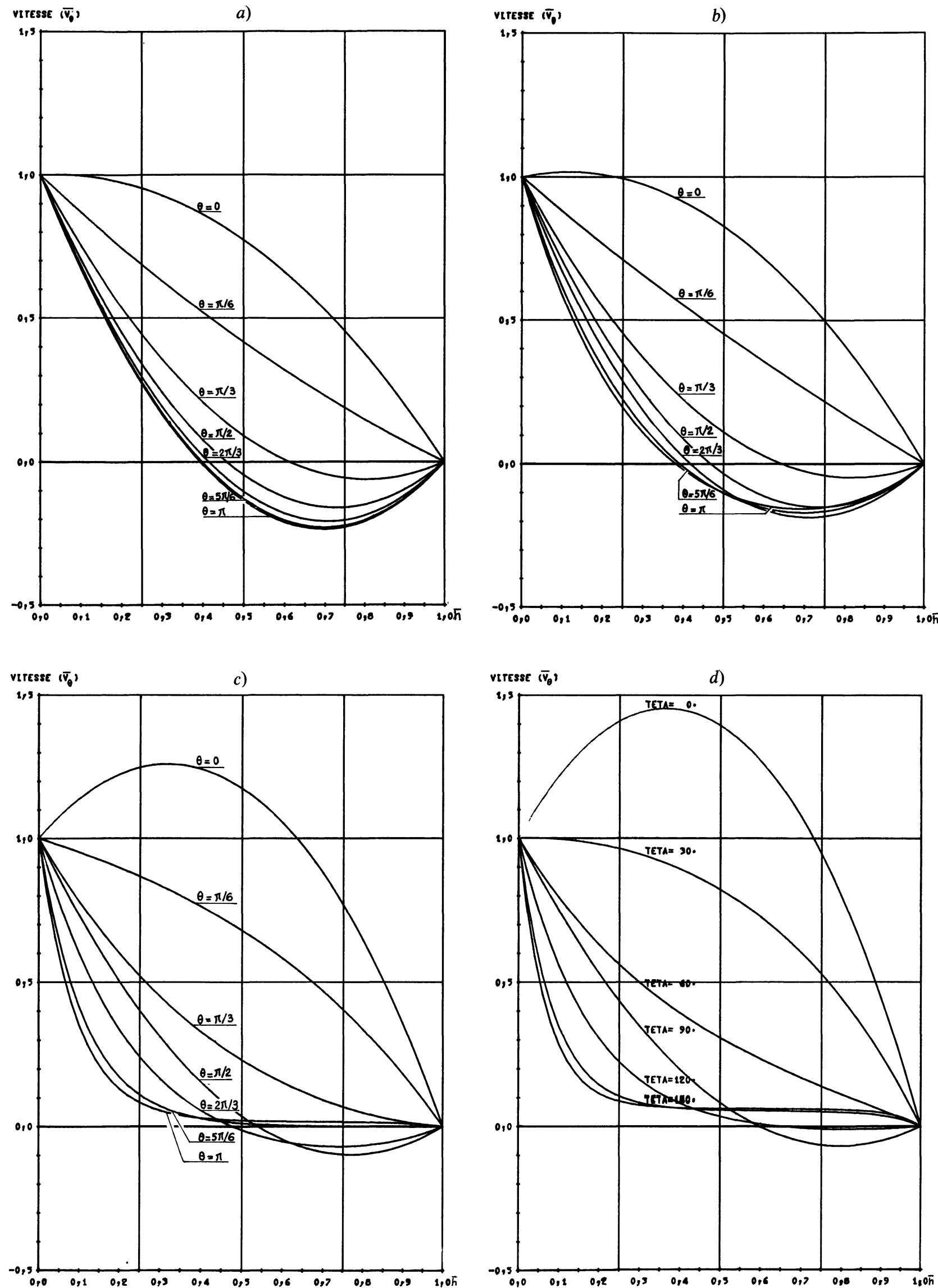

Fig. 7. - Vitesse $\bar{v}_{\theta}$, en fonction de $\bar{y}$, pour différentes valeurs de $\theta$ : a) $\left.M_{0}=0,00, \Omega t=0, \varepsilon=0,8 ; b\right) M_{0}=1,41, \Omega t=0, \varepsilon=0,8$; c) $\left.M_{0}=4,24, \Omega t=0, \varepsilon=0,8 ; d\right) M_{0}=5,66, \Omega t=0, \varepsilon=0,8$.

[Velocity $\bar{v}_{b}$, versus $\bar{y}$, for different values of : $a$ ) $M_{0}=0.00, \Omega t=0$, $\left.\varepsilon=0.8 ; b) \quad M_{0}=1.41, \Omega t=0, \varepsilon=0.8 ; c\right) M_{0}=4.24, \Omega t=0$, $\varepsilon=0.8 ; d) M_{0}=5.66, \Omega t=0, \varepsilon=0.8$.] 
et par suite

$$
\bar{F}_{Y}=-X_{2}^{\prime \prime}-\bar{q}_{\mathrm{v}}^{\prime} \int_{0}^{2 \pi} F_{1}^{\prime}(\theta) \sin \theta \mathrm{d} \theta
$$

$\left(\bar{q}_{\mathrm{v}}^{\prime}\right.$ : débit réduit, fonction de $t$ seul)

considérées maintenant comme fonctions du temps, ont des valeurs nulles et que, pour une charge extérieure de direction donnée $Y^{\prime} Y$, le palier aura une position stable définie par l'axe perpendiculaire $X^{\prime} X$.

On a représenté, à titre indicatif, les profils de vitesse (Figs. 7 à $7 d$ ) aux instants $2 k \pi / \Omega$ ( $k$ entier) dans différentes sections de la veine, pour différentes valeurs de $M_{0}$ et pour une excentricité relative de 0,8 . Sur la figure 8 , on a tracé l'enveloppe des variations de pression $[\overline{\hat{p}}(\theta, \bar{z}, \bar{t})-\overline{\hat{p}}(0, \bar{z}, \bar{t})]$ en fonction de $\theta$ pour $M_{0}^{2}=2$ et $\varepsilon=0,8$.

$\mathrm{Si}$ on remarque que, pour un métal liquide, le rapport $\sigma / \eta$ est de l'ordre de $10^{9}$ S.I., et que, dans un palier, $a$ vaut au plus quelque $0,1 \mathrm{~mm}$, le nombre de Hartmann correspondant vaut $M_{0}=\sqrt{10} \mu_{0} H_{0}$.

Excepté dans le cas d'un inducteur supraconducteur, il est difficile d'obtenir dans la veine des inductions $\mu_{0} H_{0}$ supérieures à $1 \mathrm{~T}$; aussi les valeurs pratiques du nombre de Hartmann sont-elles comprises dans l'intervalle $[0,3 \sqrt{2}=4,2]$ défini précédemment, justifiant encore le bien fondé de l'hypothèse sur les faibles fluctuations de la vitesse.

3. Etude expérimentale. - Nous avons conçu, au Laboratoire une maquette permettant de tester la théorie proposée. Plusieurs possibilités s'offrent a priori pour cette vérification :

a) Mesure du coefficient de frottement.

b) Mesure de l'excentricité relative.

c) Mesure de la résultante des forces de pression.

d) Détermination de la répartition des pressions.

Des mesures des types $a, b, c$, classiques en hydrodynamique, sont rendues impossibles ici à cause de la présence des joints d'étanchéité ; ceux-ci exercent en effet sur l'arbre une force dont nous ne connaissons ni la direction, ni le module. Le couple de frotte-

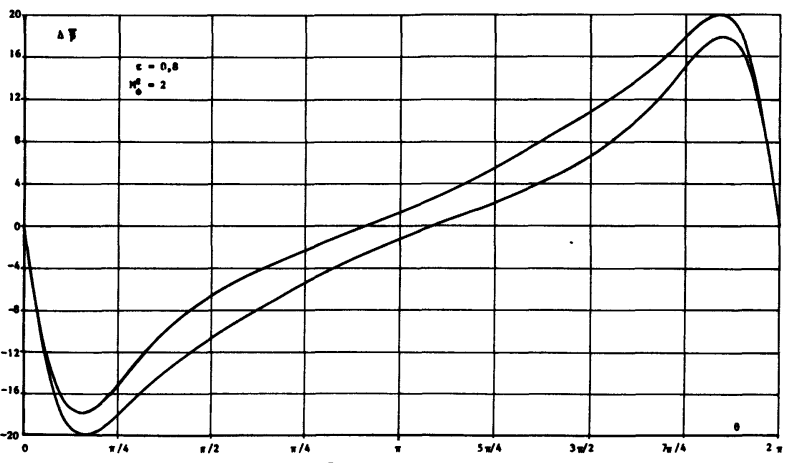

FIG. 8. - Courbes, enveloppes, en fonction de $\theta$, des variations de pressions instantanées.

[Enveloppe curves, versus $\theta$, of the instantaneous pressure variations.] ment exercé est également inconnu. Signalons simplement que cette force et ce couple sont notamment fonctions de la température et de la vitesse de rotation de l'arbre. On a donc mesuré les pressions et comparé la répartition correspondante avec celle, théorique, obtenue en traçant la courbe

$$
\begin{aligned}
& \langle\overline{\hat{p}}(\theta, \bar{z}, \bar{t})\rangle-\langle\overline{\hat{p}}(0, \bar{z}, \bar{t})\rangle=\frac{I_{2}-2 \pi M_{0}^{2}}{I_{1}} \times \\
& \quad \times \int_{0}^{\theta} F_{1}(\theta) \mathrm{d} \theta-\int_{0}^{\theta} F_{2}(\theta) \mathrm{d} \theta+M_{0}^{2} \theta+M_{0}^{2} \frac{\varepsilon}{2} \sin \theta
\end{aligned}
$$

en fonction de $\theta$.

3.1 RÉAliSATION DE LA MAQUETTE ET DISPOSITIF DE MESURE (Figs. 9 à 12). — Le système inducteur (A), composé d'une carcasse bipolaire en acier doux est $\mathrm{du}$ type inducteur tournant de machine synchrone; il est solidaire de l'arbre, qui est maintenu par deux flasques à roulements à billes (B) et a les caractéristiques suivantes :

$$
\begin{array}{ll}
\text { - diamètre extérieur } & =101,72 \pm 0,0025 \mathrm{~mm} \\
\text { - longueur totale } & =270,7 \pm 0,025 \mathrm{~mm} \\
\text { - longueur utile } & =138,2 \pm 0,025 \mathrm{~mm} .
\end{array}
$$

Les bobinages, responsables de la force magnétomotrice, sont réalisés sur les pôles et reliés aux deux bagues collectrices montées sur l'une des extrémités de l'arbre et immobilisées en rotation et en tr.ınslation.

L'ensemble est noyé dans la résine époxyde et gainé par un fourreau d'aluminium anodisé dur, dont l'épaisseur est $1,86 \pm 0,1 \mathrm{~mm}$. Cette gaine, en métal amagnétique anodisé, présente ainsi les caractéristiques nécessaires :

liquide

- d'isolation électrique entre l'arbre et la veine

- de dureté superficielle pour le frottement des joints d'étanchéité $(F)$ des flasques latéraux.

L'arbre est entraîné par une machine à courant continu dont la vitesse est réglable de 0 à $2000 \mathrm{tr} / \mathrm{min}$.

Le coussinet $(G)$ est constitué par un tube en acier doux dont l'alésage est recouvert de résine époxyde assurant son isolation électrique par rapport à la veine de métal liquide. Il a les caractéristiques suivantes :

$$
\begin{aligned}
\text { - diamètre extérieur } & =151,9 \pm 0,025 \mathrm{~mm} \\
\text { - diamètre intérieur } & \pm \text { (résine comprise) } \\
\text { - longueur } & =102,29 \pm 0,0025 \mathrm{~mm} \\
\text { - épaisseur de la } & 138,2 \pm 0,025 \mathrm{~mm} \\
\text { résine époxyde } & =0,5 \pm 0,05 \mathrm{~mm}
\end{aligned}
$$

Le dispositif manométrique $(\mathrm{J})$ est composé de quatorze tubes de verre dont deux servent à purger l'air de la veine liquide. Ces tubes sont reliés par un dispositif élastique aux douze prises de pression $(\mathrm{H})$ 


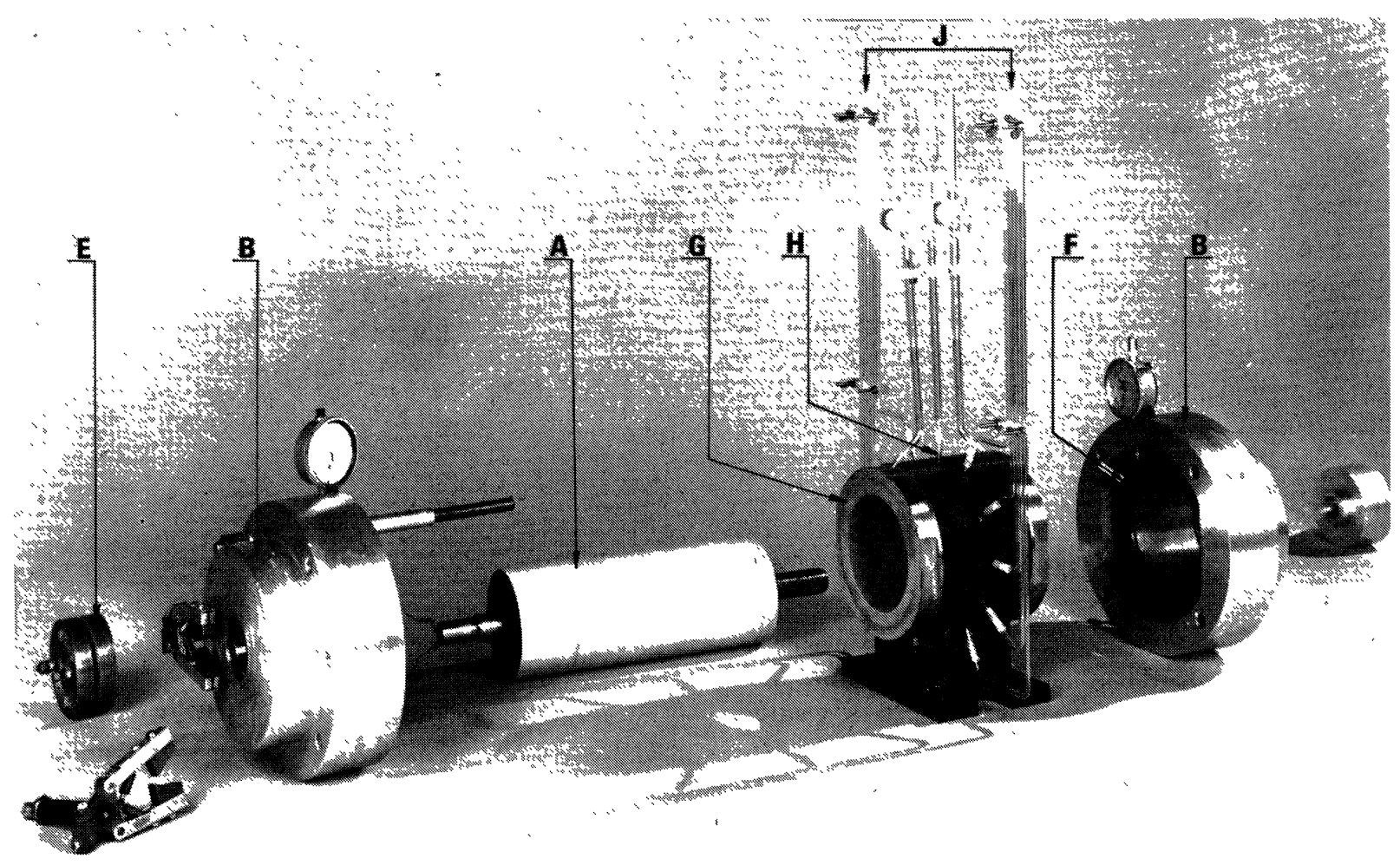

Fig. 9. - Organes du palier M.H.D.

[Components of the M.H.D. bearing.]

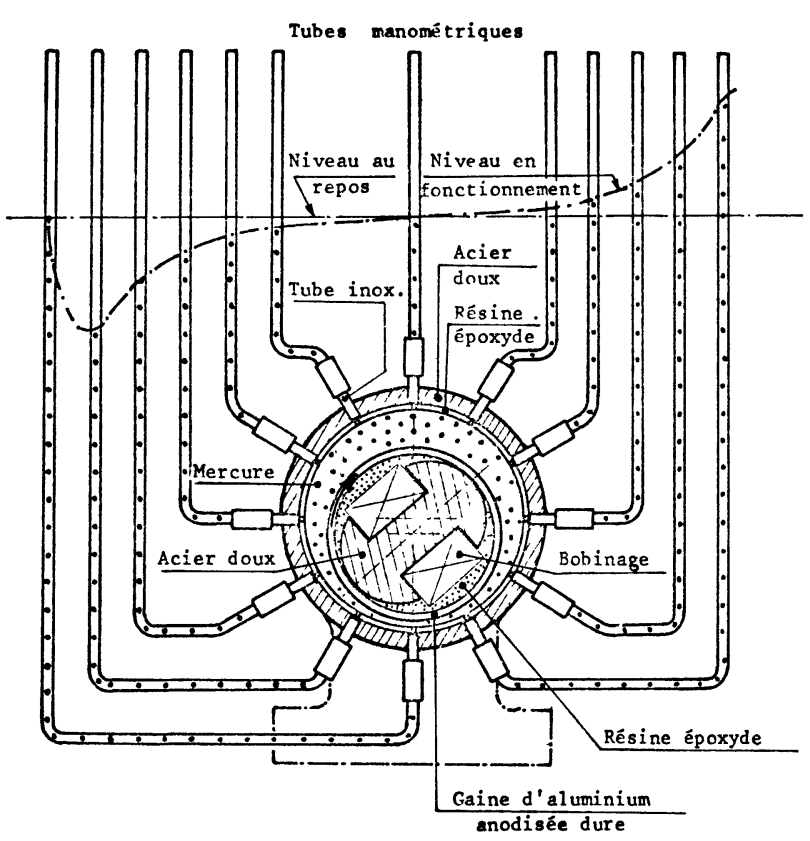

Fig. 10. - Dispositif de mesure des pressions. [Device for pressure measurements.]

équidistantes, en acier inoxydable (Figs. 9 et 10); elles sont réparties sur la périphérie de la zone centrale $\mathrm{du}$ coussinet pour éviter les perturbations dues aux effets de bords.

La dimension et la forme des trous de communication des prises de pression avec la veine liquide a été choisie de manière à éviter le plus possible les

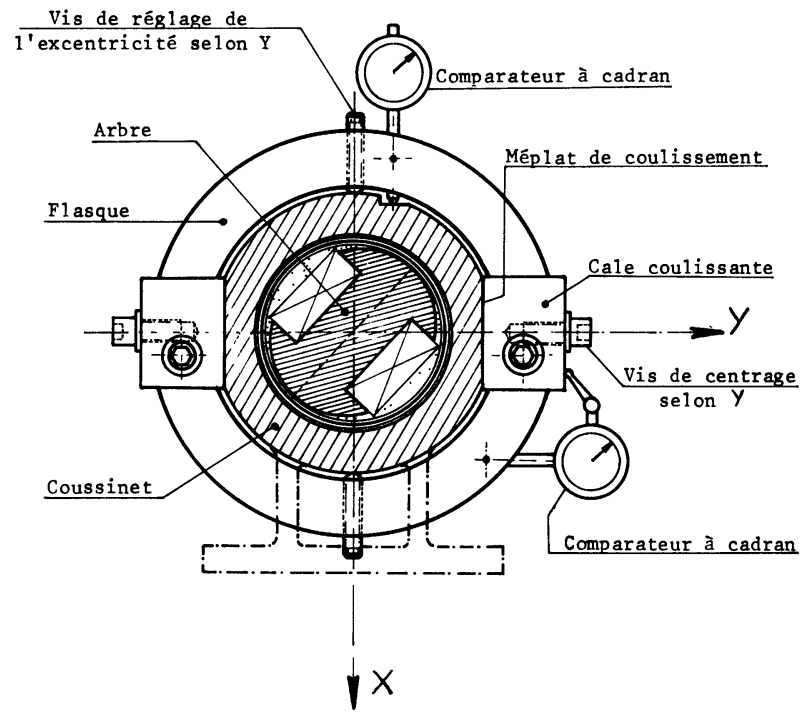

Fig. 11. - Vue en coupe de la maquette. [Cross-section of the model.]

erreurs de mesure dues à leur influence sur l'écoulement du liquide.

En prenant pour base les différentes études et essais traitant de ce sujet [21], il a été retenu un diamètre de $1 \mathrm{~mm}$ pour les orifices de communication. Il apparaît que cette dimension n'introduit pas d'erreur supérieure à $3 \%$ sur la valeur de la pression. La dénivellation du métal liquide dans chaque tube détermine la répartition de la pression en fonction 


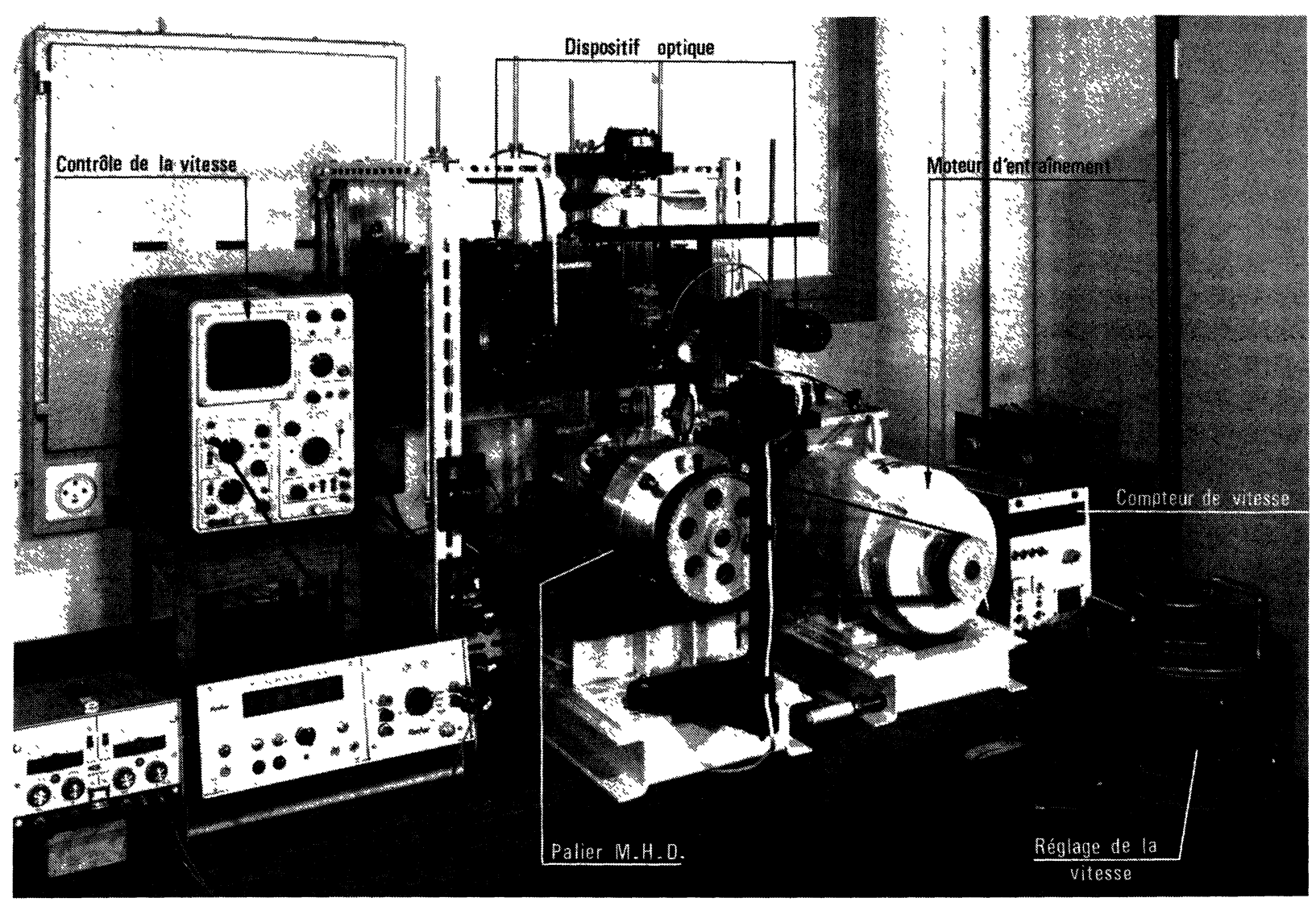

FIG. 12. - Vue d'ensemble du dispositif expérimental.

[General view of the experimental device.]

de l'angle géométrique, comme il est indiqué sur la figure 10.

Les flasques latéraux assurant l'étanchéité de la veine liquide et le positionnement de l'arbre sont en alliage amagnétique, afin de ne pas introduire de perturbation du champ magnétique aux extrémités du palier.

Ces deux flasques possèdent un dispositif de réglage de l'excentricité de l'arbre par rapport au coussinet et un dispositif de contrôle de ce réglage.

Le réglage peut se faire selon l'axe horizontal (axe $Y$ ) et selon l'axe vertical (axe $X$ ), ceci afin de rattraper les défauts géométriques de fabrication et d'assemblage du palier magnétohydrodynamique. La partie de chaque flasque en contact avec la veine conductrice est recouverte d'une peinture époxyde qui assure son isolation thermique. De plus, la veine est rendue étanche par l'insertion d'un joint torique dynamique en caoutchouc entre l'arbre et le flasque et d'un joint statique entre le coussinet et le flasque.

Pour des raisons de facilité de mise en œuvre, le mercure très pur a été choisi comme métal liquide lubrifiant (mercure R.P. No 2535829 Prolabo). Les caractéristiques physiques du mercure, pour la température d'essai $t_{\mathrm{a}}$ de $19,5^{\circ} \mathrm{C}$ sont respectivement :

- la masse volumique (formule de Chappuis)

$$
\rho_{19,5}=13643 \mathrm{~kg} / \mathrm{m}^{3}
$$

- la viscosité dynamique (formule de Lacas)

$$
\eta_{19,5}=1,558 \times 10^{-3} \mathrm{P} 1
$$

- la conductivité (formule de Jaeger et Steinwehr)

$$
\sigma_{19,5}=1042868 \Omega^{-1} \mathrm{~m}^{-1} .
$$

3.1.1 Etalonnage en régime hydrodynamique. Pour des raisons de facilité de mise en œuvre, nous avons utilisé de l'eau additionnée de Teepol, afin de graisser les joints dynamiques d'étanchéité. Cet étalonnage a permis de vérifier le bon fonctionnement de notre dispositif; en effet :

- il n'y a pas d'effet d'extrémité visible,

- les variations de pression dues aux défauts géométriques sont négligeables,

- les essais confirment la validité de la théorie proposée pour le palier en régime hydrodynamique.

3.1.2 Vitesse critique de transition. - La théorie proposée suppose que le nombre de Reynolds modifié $R_{\mathrm{e}}^{*}$ prend des valeurs négligeables devant l'unité, ce qui signifie, en régime hydrodynamique, que l'écoulement est laminaire, non tourbillonnaire.

Des mesures ont été effectuées afin de déterminer la vitesse critique caractérisant le début du régime tourbillonnaire en hydrodynamique.

A différentes vitesses et pour une excentricité relative de 0,8 , les relevés des distributions de pressions 
réduites se superposent (aux erreurs de mesure près) jusqu'à une vitesse évaluée à $52 \mathrm{tr} / \mathrm{min}$., ce qui correspond à une vitesse linéaire de l'arbre de $0,28 \mathrm{~ms}^{-1}$ et à un nombre de Taylor

$$
T=\frac{\rho}{\eta} \Omega a r \sqrt{\frac{a}{r}}=51,3 .
$$

La hauteur de dénivellation maximale dans les tubes de mesure est alors de $30 \mathrm{~mm}$, ce qui correspond sur l'écran à une hauteur de $42 \mathrm{~cm}$. Au-delà de cette vitesse, les pressions réduites croissent systématiquement. On interprète ce fait en supposant l'apparition de tourbillons de Taylor à partir d'une rotation de $52 \mathrm{tr} / \mathrm{min}$., ce qui est cohérent avec les travaux de J. Frene [18]; en effet, pour $\varepsilon=0,8$, la transition est prévue pour $T \approx 50$.

3.2 Relevés eXPÉRImentaux. - Les différents résultats d'essais, effectués avec le mercure, sont portés sur les figures 13 à 17 . Chaque point expérimental représente la moyenne des mesures faites sur une dizaine d'essais avec l'excentricité relative $\varepsilon$ choisie et une vitesse de rotation de l'arbre imposée.

Comme les mesures sont très délicates à exécuter, on a, pour chaque expérimentation, relevé l'accroissement de pression en régime hydrodynamique et en régime magnétohydrodynamique, ce qui correspond toujours à un effet différentiel significatif.

a)

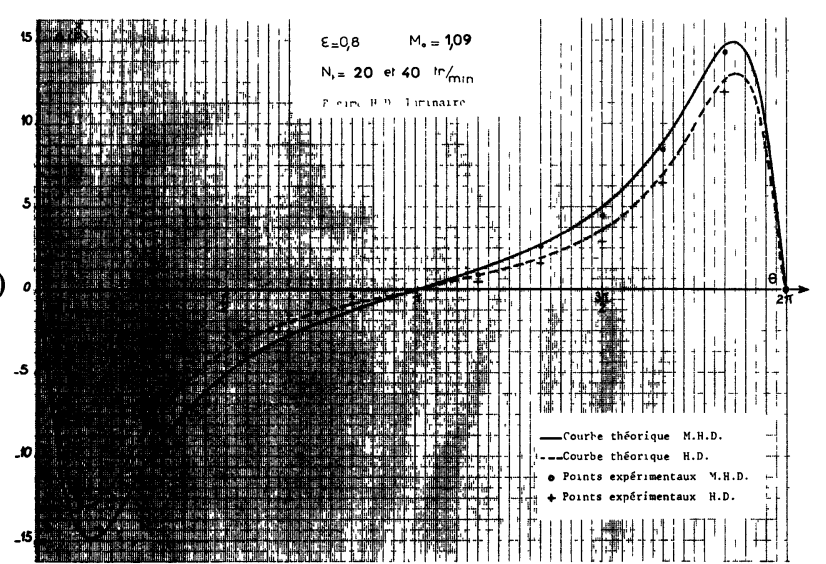

b)

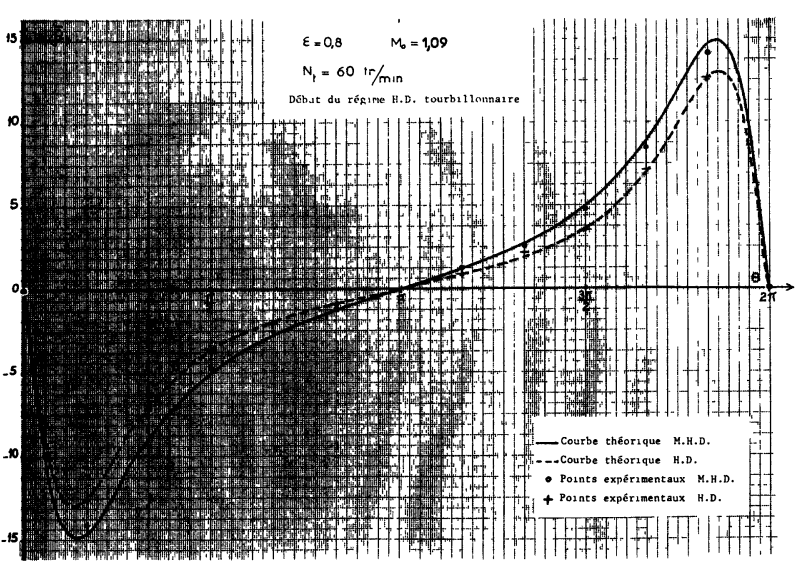

FIG. 13.
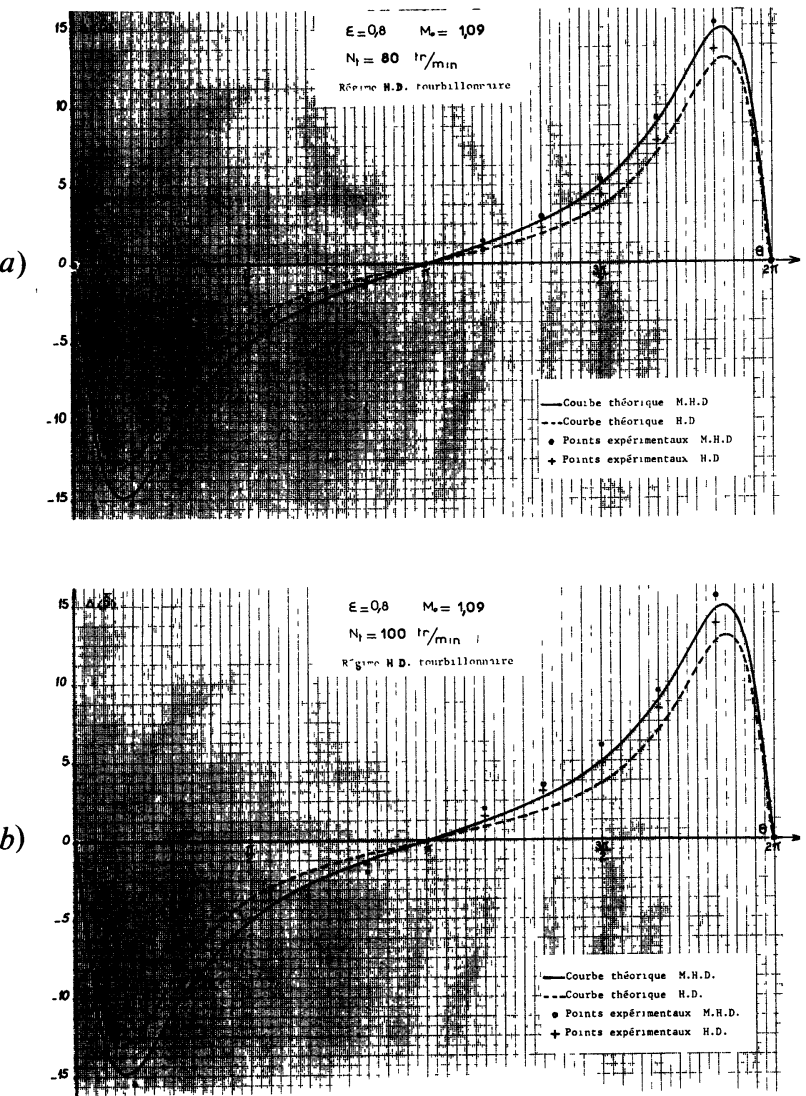

Fig. 14.
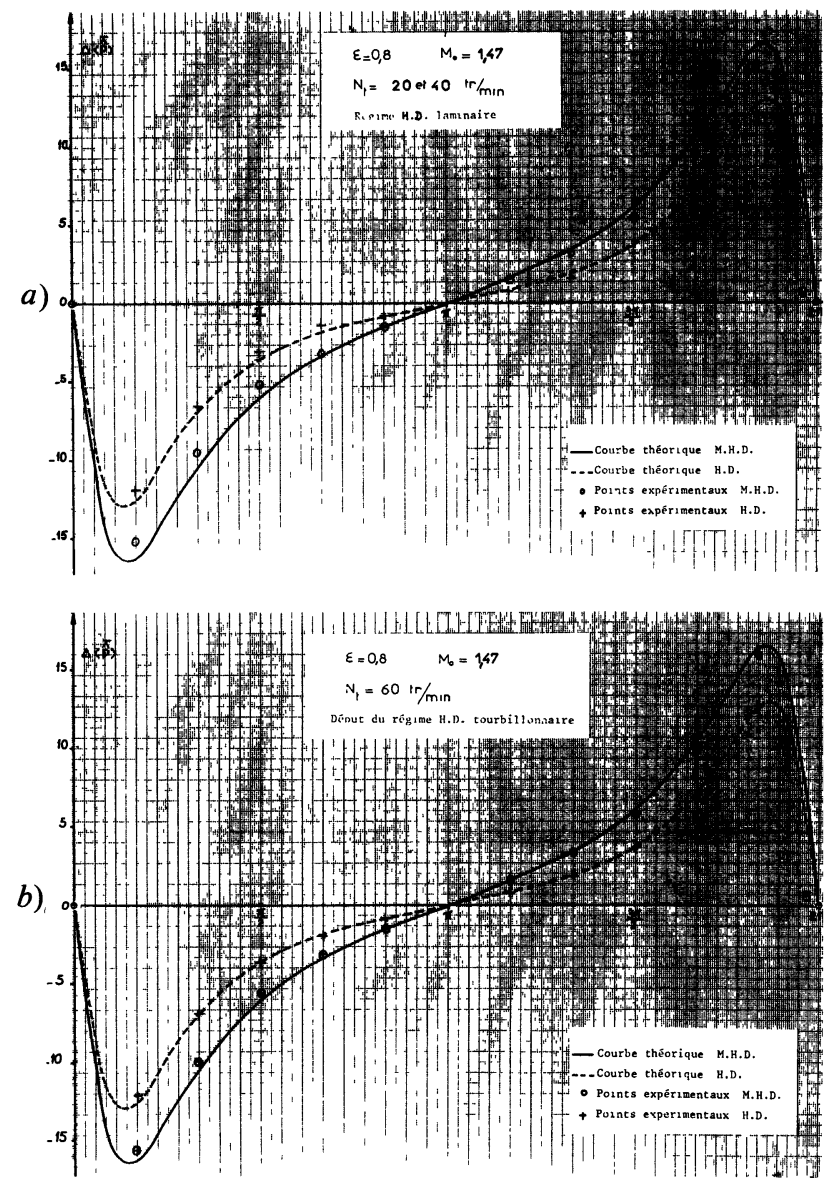

FIG. 15. 
a)

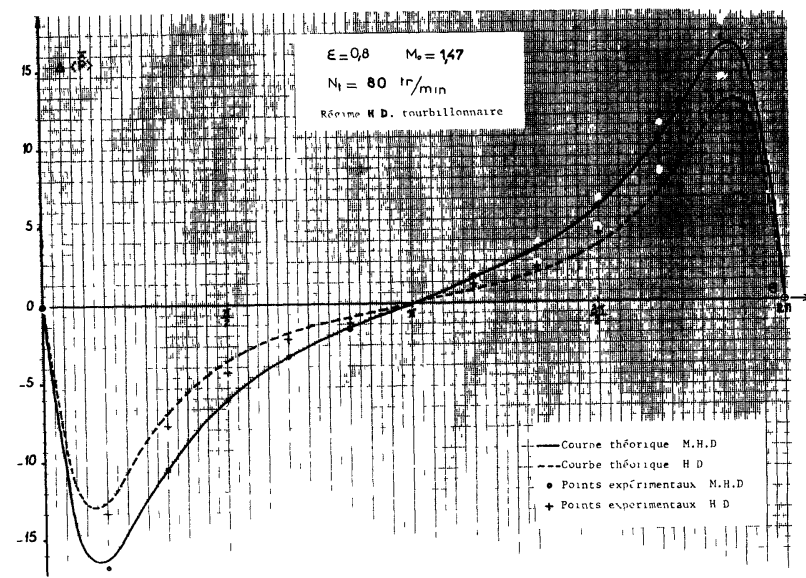

b)

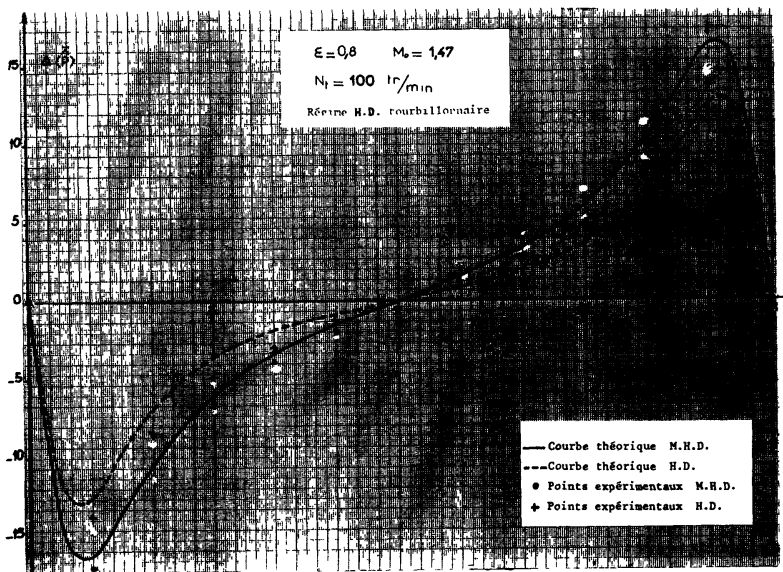

FIG. 16.

a)

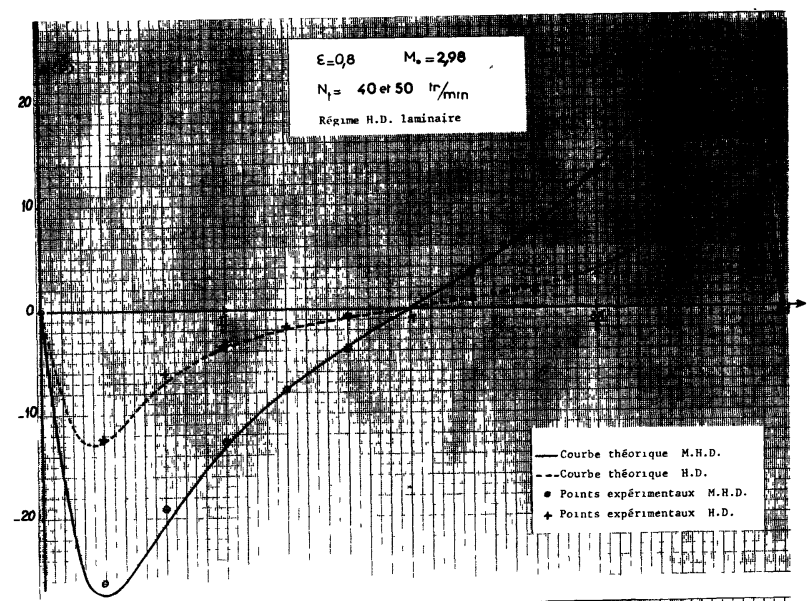

b)

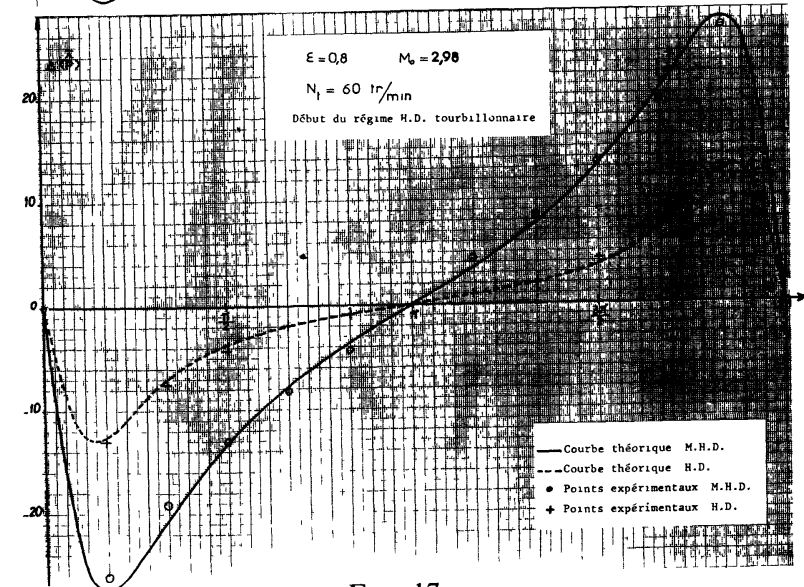

FIG. 17.
A chaque essai M.H.D., la valeur de l'induction dans la veine est relevée à l'aide d'une sonde à effet Hall. La détermination de la valeur efficace du fondamental permet ensuite le calcul du nombre de Hartmann correspondant à l'essai; rappelons que, les calculs théoriques ont été effectués en supposant une répartition sinusoïdale de l'induction dans la veine.

3.3 DisCuSSION DES RÉSUlTATS. - On constate, pour le fonctionnement du palier à des vitesses correspondant, en hydrodynamique, au régime laminaire non tourbillonnaire, une superposition des distributions de pressions réduites et une différence par défaut entre les courbes expérimentales et théoriques.

Ces écarts peuvent s'expliquer par :

- les erreurs de mesures dues à la capillarité du métal liquide

- l'incertitude sur l'excentricité relative

- la forme simplifiée de la répartition du champ magnétique employé pour le calcul de $M$.

- le fait d'avoir négligé l'accélération convective : $R_{\mathrm{e}}^{*}$ est ici de l'ordre de quelques unités $\left(R_{\mathrm{e}}^{*}=3,8\right.$ pour $r \Omega=2,8 \mathrm{~ms}^{-1}$ ) et donc non négligeable devant l'unité.

Il est intéressant de noter que le défaut par rapport à la théorie se présente en régime hydrodynamique ainsi qu'en régime magnétohydrodynamique, ce qui amène à penser que la dernière explication est probablement la bonne. L'écart relatif, plus important en M.H.D., s'interprète en remarquant qu'apparaît alors en plus l'influence du terme $\rho \frac{\partial \mathbf{v}}{\partial t}$, qui est, par rapport au terme $\eta \bar{v}_{\theta, y y}$ de l'ordre de $R_{\mathrm{e}}^{*} \frac{\tilde{V}^{*}}{r \Omega}$, c'est-àdire de quelques dizaines.

Les résultats concernant la mesure de la répartition de l'induction dans la veine sont représentés sur la figure 18 . On constate la présence d'un certain nombre d'harmoniques et d'une composante homopolaire, due à l'excentricité du palier. Négligeant cette composante homopolaire, on a calculé le nombre de Hartmann à partir de la valeur efficace de la composante pulsatoire du champ et on a recherché, à l'aide de l'ordinateur, la nouvelle répartition de pression.

Figs. 13, 14, 15, 16, 17. - Variations de pression, en fonction de $\theta$, pour différentes vitesses de rotation, avec

$$
\begin{array}{ll}
\text { Figures } 13 \text { et } 14 M_{0}=1,09 \\
\text { Figures } 15 \text { et } 16 & M_{0}=1,47 \\
\text { Figure } 17 & M_{0}=2,98
\end{array}
$$

[Pressure variations, versus $\theta$, for different speeds of rotation, with :
Figures 13 and $14 M_{0}=1.09$

Figures 15 and $16 M_{0}=1.47$

Figure $\left.17 \quad M_{0}=2.98\right]$

REVUE DE PHYSIQUe APPLIQUÉE. - T. 13, No 10, octoBRe 1978 


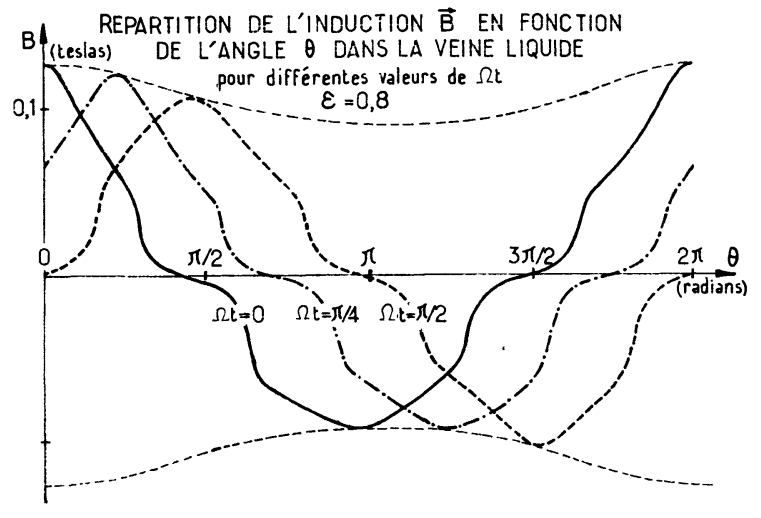

FIG. 18. - Répartition de l'induction magnétique dans la veine liquide.

[Distribution of the magnetic flux density in the liquid channel.]

Ces résultats permettent de conclure que l'influence des harmoniques majore les calculs théoriques comme indiqué ci-dessous.

Influence des harmoniques de l'induction magnétique dans les zones d'écarts de : Régime pression maximale pression minimale

$$
\text { M.H.D. } \quad 3 \% \quad 1 \text { à } 6 \%
$$

La prise en compte des harmoniques augmente donc les écarts entre les courbes théoriques et expérimentales.

Toutefois, on peut déduire des relevés expérimentaux que l'accroissement dû à l'effet M.H.D. est assez bien décrit par la théorie proposée. On constate par ailleurs que, pour des vitesses correspondant au début du régime tourbillonnaire en hydrodyna- mique, les résultats expérimentaux sont assez proches des courbes théoriques.

Indiquons pour conclure qu'il aurait été intéressant d'étudier expérimentalement les phénomènes lorsque, pour une excentricité donnée :

- le nombre de Hartmann croît, la vitesse étant maintenue constante

- la vitesse croît, le nombre de Hartmann restant constant.

La structure même de la maquette, limitant $M_{0}$ à 4 , interdit toute recherche dans la première voie. On peut toutefois constater, sur la figure 7, que l'écoulement tend à s'effectuer alors en filets quasi-parallèles, les profils des vitesses ne traversant plus de zone négative. Ce résultat suppose que l'on a toujours des fluctuations faibles de la vitesse, ce qui n'est pas nécessairement le cas. Une discussion plus complète devrait faire intervenir le paramètre d'interaction $N=M_{0}^{2} / R_{\mathrm{e}}^{*}[22]$.

Une étude préliminaire dans la deuxième direction a donné lieu à des observations curieuses. Tant que la vitesse reste inférieure à la vitesse critique $\Omega_{\mathrm{c}}$ de l'écoulement hydrodynamique d'apparition des tourbillons de Taylor, on observe (Figs. 13a, 15a et $17 a$ ) qu'il y a superposition des répartitions de pression respectives en H.D. et en M.H.D. Dès que la vitesse dépasse le seuil $\Omega_{\mathrm{c}}$, il y a alors décollement de ces répartitions vers le haut (Figs. 14, 16 et 17b) aussi bien en H.D. qu'en M.H.D. On interprète ce fait en supposant que le champ, pulsant au point de vue du métal liquide, ne lamine pas l'écoulement au sens strict, c'est-à-dire sans apparition de tourbillons. Il semblerait qu'il existe en M.H.D., dans ce cas précis, un régime secondaire dont la vitesse de transition serait la même qu'en hydrodynamique.

\section{Bibliographie}

[1] Stahlhuth, P. H., Trippett, R., Liquid Metal Bearing Performance in Laminar and Turbulent Regimes. ASLE Trans. 5 (1962) 427-436.

[2] Burton, R. A., Carper, H. J., Fundamental investigation of liquid Metal lubricated journal bearings, Southwest Research Institute 1228-26 juillet 1964

[3] DeCKer, O., ShImP, G., Mercury-lubricated hybrid bearings for the silent compact auxiliary power (S.C.A.P.) system, A.S.L.E. Trans. 8 (965) 357-366.

[4] Schuller, F., Anderson, W., Nemeth, Z., Experiments with hydrodynamic Journal bearings of various materials and designs in sodium at temperatures to $800 \mathrm{~F}, A S L E$ Trans. 11 (1968) 140-154.

[5] Moris, B., Quelques aspects du frottement en sodium liquide. G.A.M.I., I.S.M.C.M. Journées d'Etudes des 20 et 21 mars 1968.

[6] Bertinov, A. I. et Metun, V. B. (En Russe) Palier magnétohydrodynamique, Magnit. Gidrodinam, S.S.R., 1969, $\mathrm{n}^{\circ} 2$, p. 3-18.

[7] Hughes, W. F., ElCo, R. A., M.H.D. Journal bearing, A.R.S. Journal 05/1962, p. 776-778.

[8] Kuzma, D. C., The magnetohydrodynamic Journal bearing, Journal of Basic Engineering, Trans. ASME, series D, 85 (1963) 424-428.
[9] Kuzma, D. C., The finite magnetohydrodynamic Journal bearing, Journal of Basic Engineering, Trans. ASME, series D, 86 (1964) 445-448.

[10] Dudzinsky, S. H., Young, F. J. and Hughes, W. F., On the load capacity of the magnetohydrodynamic journal bearing, Journal of Lubrication Technology, Trans. $A S M E, 90$ (1968) 139-144.

[11] KamiYama, S., Magnetohydrodynamic journal bearing (Report 1), Journal of Lubrication Technology, Trans. $A S M E$, series F, 91 (1969) 380-386.

[12] Barral, J., Contribution à l'étude des paliers magnétohydrodynamiques. Thèse de Doctorat $3^{\mathrm{e}}$ cycle, Université Paris-VI, 1973.

[13] Barral, J., Kant, M., General study of electromagnetic bearings. Trans. I.E.E.E. (Magnetics). Mag-11, 09/1975.

[14] Sasada, T., Kurosaki, Y., Honda, K., Kamijo, K., M.H.D. Journal bearing in a magnetic field perpendicular to its axis (1st report analysis of an infinitely long bearing). Bull. JSME 17 (1974) 1645-1651.

[15] Sasada, T., Kurosaki, Y., KamiJo, K., M.H.D. Journal bearing in a magnetic field perpendicular to its axis (2nd report-experiment with mercury), Bull. JSME 17 (1974) $1652-1656$ 
[16] SChoenhenz, D., Contribution à l'étude des paliers M.H.D. à champ tournant. Mémoire C.N.A.M. Paris 1977.

[17] Fediaevsky, C., Voitkounski, I. et Fadeev, Y., Hydromécanique, traduit du Russe, Moscou, Mir, 1974.

[18] Frene, J., Contribution à l'étude des régimes de transition dans les paliers lisses. Thèse Docteur-Ingénieur, Lyon, 1970.

[19] DeLPhiN, P., Les paliers hydrodynamiques en régime laminaire et non laminaire. Mémoire C.N.A.M. Lyon, 1973.
[20] Kant, M., Barral, J., Bonnefille, R., Sur la définition d'un coefficient de transfert des transmetteurs électrodynamiques. C.R. Hebd. Séan. Acad. Sci. 285, série B, 81-83.

[21] Barat, M., Les mesures de pression à l'aide de prises à la paroi, C.E.A., E.D.F., Cycle de conférences sur les techniques de mesure dans les écoulements, 24-25 septembre 1973, p. $245-250$.

[22] Alemany, A., Moreau, R., Ecoulement d'un fluide conducteur de l'électricité en présence d'un champ magnétique tournant, Journal de Mécanique no 4, 1977-1978, vol. 16. 